\title{
Hybrid Field Theory and Particle Simulation Model of Polyelectrolyte-Surfactant Coacervation
}

\author{
Jason J. Madinya and Charles E. Sing* \\ Department of Chemical and Biomolecular Engineering, University of Illinois at Urbana \\ Champaign, 600 S. Matthews Ave., Urbana, IL, USA. \\ E-mail: cesing@illinois.edu
}

\begin{abstract}
Solutions of oppositely-charged polyelectrolytes and surfactants have been widely studied for a variety of applications; they play an important role in the formulation of personal care products, can be used as an effective strategy for drug encapsulation, and serve as analogues to biological systems such as biomolecular condensates. Surfactant molecules self-assemble into micellar macroions that are known to form complexes with oppositely-charged polyelectrolytes, and can undergo a bulk liquid-liquid phase separation known as complex coacervation. This process results in a 'coacervate' phase that is rich in macroions, and a 'supernatant' phase that is dilute in macroions. It is challenging to model this phase separation process, due to the disparate length scales and strong Coulombic interactions in these mixed macroion systems. In this work, we present a hybrid simulation and field theory model to describe polyelectrolytes/surfactants solutions, where the surfactant species has self-assembled into worm-like micelle structures. We use self-consistent field theory (SCFT) to model the polyelectrolytes in the solution
\end{abstract}


which interact with the surfactant micelles. The surfactant micelle structures are determined by performing Monte Carlo (MC) simulations, which are used to determine applied external fields in the SCFT portion of the model. We use these calculations to determine the system free energy and map the phase diagrams for polyelectrolytesurfactant coacervates, and subsequently consider the effect of a number of molecular parameters such as polyelectrolyte chain length, the volume of the interacting micelle surface-sites, and the electrostatic binding energy between the polyelectrolyte and micelle surface. Our model shows that local charge-charge correlations are critical for phase separation to occur. Additionally, we evaluate the statistics of micelle bridging by the polyelectrolyte, and the relationship between bridging and the densities of the macroions and salt ions. This hybrid SCFT/MC model can be generalized to study a variety of mixed macroion systems, and make predictions for phase behavior and molecular structure.

\section{Introduction}

Solutions of oppositely charged polyelectrolytes and surfactants have been studied extensively for a variety of applications, including drug encapsulation, ${ }^{1-3}$ personal care products, ${ }^{4-8}$ and food products. ${ }^{9-11}$ These systems are known to exhibit a number of self-assembly or phase behaviors, driven by a complicated interplay of various intermolecular forces, particularly hydrophobic and electrostatic interactions. ${ }^{12-17}$ Hydrophobicity drives the self-assembly of surfactant molecules into micelle structures, ${ }^{18,19}$ and both hydrophobic and electrostatic interactions can promote the complexation of the polyelectrolytes with the oppositely-charged surfactants. ${ }^{16,17}$ In addition to the competition between these interactions, the multicomponent nature of these systems - which consists of the polyelectrolyte, the surfactant molecules, both cation and anionic salt species, and water - gives rise to a number of desirable properties that can be readily tuned. ${ }^{20-22}$ Most importantly, phase behavior and self-assembly give rise to rheological properties such as foamability, wetability, and lubrication that are commonly 
used to engineer the sensorial properties ${ }^{8}$ important for consumer applications (i.e. personal care and food products). Despite the key industrial role of this class of materials, there remain significant gaps in the community's knowledge of the fundamental physics governing the phase behavior of polyelectrolyte-surfactant mixtures.

Most of the current understanding of polyelectrolyte-surfactant mixture phase behavior stems from experimental measurement, and typically involves characterizing a number of key quantities that capture the relationship between micelle formation, electrostatic complexation, and assembly. ${ }^{12,23,24}$ In aqueous surfactant solutions, the critical micelle concentration $(\mathrm{CMC})$ is one such key quantity, and is the concentration above which the surfactant molecules self-assemble into micelle structures. ${ }^{24}$ For charged surfactants in solution with oppositely charged polyelectrolytes, it is known that this is supplanted by a critical aggregate concentration $(\mathrm{CAC})$ required for the surfactants to aggregate into micelle structures. This concentration is lower than the CMC, because the interactions between the polyelectrolyte and the surfactant counteracts the like-charge repulsive forces due to the aggregation of the surfactant charges. ${ }^{23,25}$ This results in the surfactants forming aggregates (i.e. complexes) with the polyelectrolytes, which decorate the charged surfaces of the aggregates; these complexes form the foundation for a number of phases and self-assembled structures, and represent the major challenge for describing polyelectrolyte-surfactant systems. ${ }^{26,27}$

One feature of many polyelectrolyte-surfactant systems is the emergence of a bulk phase separation into a concentrated liquid phase known as a coacervate and a dilute liquid phase known as a supernatant. ${ }^{28-31}$ This process, known as complex coacervation is thus a liquidliquid phase separation observed in any number of macroion systems, ${ }^{32}$ but is particularly well-studied in solutions comprising of oppositely-charged polyelectrolytes. ${ }^{32-54}$ Despite the ubiquity of polyelectrolyte-polyelectrolyte coacervation in the literature, there has recently been considerable interest in understanding complex coacervation in mixed macroion solutions; this includes solutions of polyelectrolytes and oppositely-charged surfactants, ${ }^{28-31,55}$ which is the primary focus of this paper, but also solutions of charged colloids and pro- 
teins. ${ }^{56-58}$

In addition to complex coacervation, polyelectrolyte-surfactant systems above the CAC can self-assemble into structures formed by ordering the surfactant micelles. ${ }^{26,27} \mathrm{~A}$ number of structures have been proposed and measured, including precipitates ${ }^{59,60}$ and gels. ${ }^{61,62}$ More standard self-assembled structures, such as lamellar structures, ${ }^{63}$ as well as cylindrical micelles and spherical vesicles ${ }^{64-66}$ have been reported, where the polyelectrolyte chains forms a network with the cylindrical micelles or spherical vesicles as the nodes. The spherical vesicle structure with the polyelectrolytes connecting the vesicles is often referred to as 'string of pearls' or 'necklace' structure in the literature. ${ }^{64-66}$ Additionally, core-shell structures have also been reported, where there is a critical polymer-surfactant charge ratio $Z_{c}$, above which the surfactants form a micellar core with the polylectrolytes decorating the surface of the core. ${ }^{66,67}$

While there has been extensive work on characterizing these polyelectrolyte-surfactant systems, there is still not an agreed-upon physical picture of phase separation and selfassembly in polyelectrolyte-surfactant systems and other mixed macroion solutions. Works by Dubin et. al, ${ }^{68}$ Wang et. al $^{14}$ and Kizilay et. al., ${ }^{69}$ show that polyelectrolyte-surfactant complexation and coacervation is highly dependent on the linear charge density of the polyelectrolyte, the surface charge density of the surfactant-micelle, and the ionic strength of the solution. Wang et. al. ${ }^{14}$ suggests that changes in salt concentration can enhance or suppress coacervation due to changes in the binding affinity between the polymer and micelle. These findings suggest that strong electrostatic attractions between the oppositely-charged macroions are a driving force in complexation and coacervation. These electrostatic attractions can drive phase separation via several mechanisms, depending on the molecular details of the interacting macroions; one driving force for coacervation is a phenomena known as 'counterion release', which is characteristic of macroions with high charge densities. ${ }^{70,71}$ Counterion release refers an increase in translational entropy due to the release of condensed salt ions ${ }^{70}$ upon complexation between two oppositely charged macroions, ${ }^{71}$ and has been 
proposed as the key mechanism underpinning the coacervation of two polyelectrolytes. ${ }^{36}$ Counterion release and the related concept of ion pairing are invoked in a number of models that are developed to explain a variety of coacervates composed of high linear charge density polyelectrolytes, and are used to explain not just standard polyelectrolyte coacervation, ${ }^{37}$ but also charge-sequenced polyelectrolytes, ${ }^{72}$ as well as self-coacervation in sequenced polyampholytes. ${ }^{73}$ Conversely, low linear charge density polyelectrolytes are thought to form coacervates due to fluctuation-driven attractions, an approach that has also been used to model sequence and polyampholyte effects. ${ }^{36,47,49,74-76}$ The relationship between fluctuationdriven and counterion relase-based mechanisms, and their role in real polyelectrolyte coacervates, remains an active area of research. In coacervation between polyelectrolytes and oppositely-charged surfactant-micelles, coacervation may be driven by electrostatic fluctuation forces and/or counterion release, however the impact of one driving force over the other is thought to depend on the specific macroion species being studied. ${ }^{77}$ Efforts to understand these thermodynamic mechanisms has focused on the strong dependence of phase behavior on both temperature ${ }^{78}$ and polyelectrolyte molecular weight. ${ }^{79}$

There have been very few efforts to model mixed macroion coacervates with respect to either ion-piaring or fluctuation-based attraction, despite significant progress in polymeric coacervates. Perhaps the only theoretical or computational effort we are aware of has been the progress made by Ganesan and coworkers to model fluctuation-based attraction between charged colloids in polyelectrolyte solutions. ${ }^{80-84}$ They have modeled both uniformly charged colloids, ${ }^{80,84}$ as well as patchy colloids, ${ }^{81-83}$ and have quantified their attraction in oppositelycharged polyelectrolyte solutions. This has served as the input to larger length-scale simulations of coacervation, and they have mapped out some important phase behaviors. Like the linear chain systems, however, fluctuation-driven attraction is typically limited to small concentrations of charged species species (i.e. salt, polymer, and micelle). This is because 'ion pairing' and other local correlation interactions are not as clearly resolved. In analogy to polyelectrolyte coacervates, ${ }^{43,85,86}$ a model to account for ion pairing and other local cor- 
relation effects will be necessary to span all polymer and micelle charge densities found in experimental systems.

In this work, we present a field theory model for coacervation in oppositely-charged surfactant-micelle and polyelectrolyte solution. This model incorporates ion pairing ideas developed in our prior work on polyelectrolyte-polyelectrolyte coacervation, ${ }^{37,72,73,87-90}$ while still resolving the conformational attributes of these polyelectrolyte chains as they bridge and interact with surfactant micelles. To account for these micelles, we use results from Monte Carlo simulations of worm-like micelle structures to determine the external potentials acting upon the polyelectrolytes in solutions. We show that correlation-driven, electrostatic interactions between the polyelectrolyte and surfactant-micelle are required to exhibit coacervation in this mixed macroion solution. We map phase behavior in the context of 'effective' ternary phase diagrams used in works by Svensson, et. al. ${ }^{31,91}$ Our model is consistent with a number of features present in these phase diagrams; we predict associative coacervate formation in surfactant-micelle and polyelectrolyte solutions, ${ }^{30}$ meaning both macroions separate into the same phase. We also show that the addition of simple salt results in a reduction of the two-phase region and at a critical concentration of added salt, phase separation no longer occurs. We are able to explore the effect of a number of molecular parameters, including polyelectrolyte chain length, surface site charge density, and the strength of the electrostatic correlations between micelles. These parameters are related to a number of quantities commonly used to manipulate phase behavior in polyelectrolyte-surfactant com-

plexes, such as surfactant tail length, ${ }^{92,93}$ polymer molecular weight, ${ }^{92,94}$ polymer backbone hydrophobicity, ${ }^{95}$ and charge density and charge distribution in the polyelectrolyte. ${ }^{96-98}$

\section{Hybrid Monte Carlo and Field Theory Model}

Our approach to modeling polyelectrolyte-surfactant micelle coacervation relies on a hybrid approach to capture both the structure of the surfactant micelle components, as well as the 


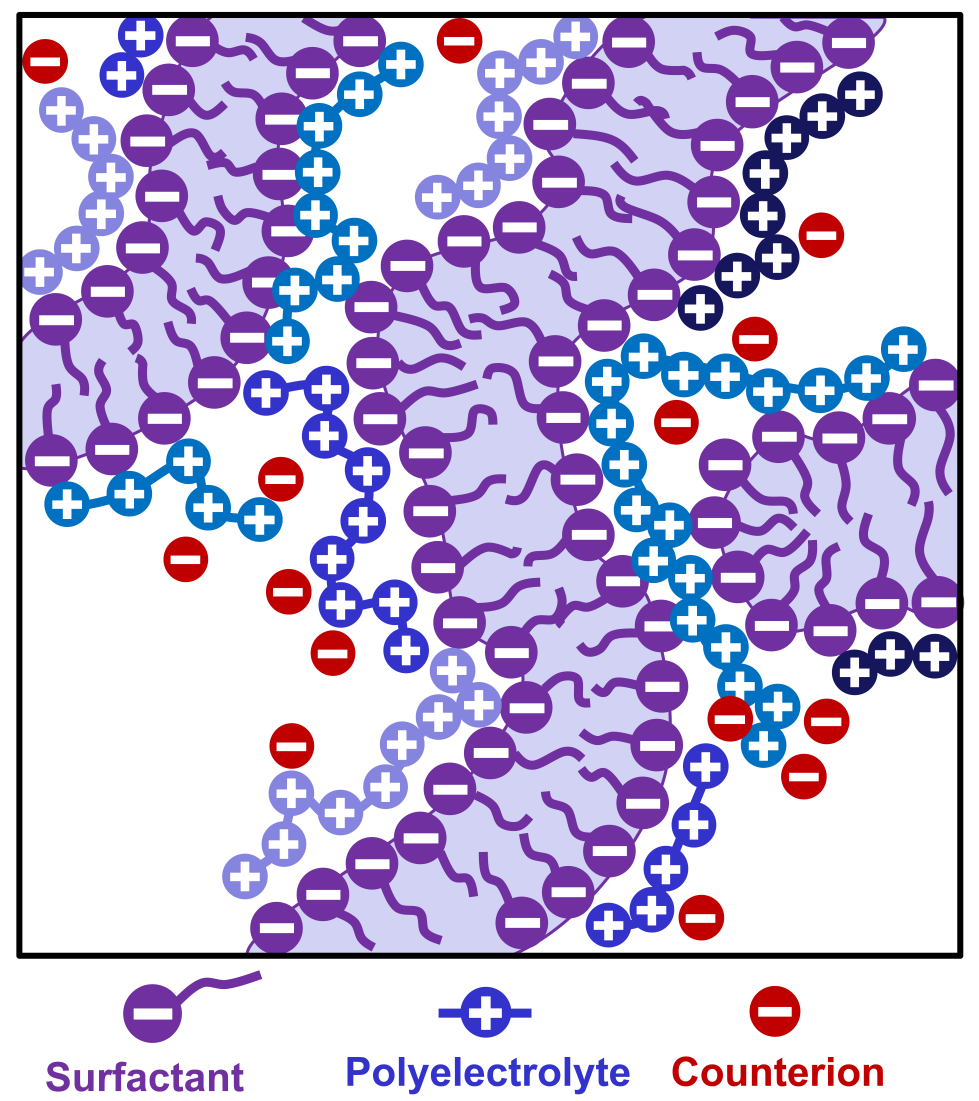

Figure 1: Schematic illustrating the polyelectrolyte and surfactant-micelle solution. The polyelectrolyte chains are shown in blue, where different shades represent individual chains. Small ion salt molecules are represented by the red spheres. The surfactant-micelles are represented by the purple structures, where the surfactant molecules are the individual sets of heads and tails, and they are organized into a worm-like micelle structure, with the charged heads on the surface of the micelle. The polyelectrolyte chains are shown interacting with, and binding to the micelle surfaces, where a single polyelectrolyte chain may be binding with separate micelle structures. 
conformations of the complexing polyelectrolytes. We consider a system comprised of cylindrical surfactant micelles with negatively-charged surfaces, positively-charged polycations, and small molecule salt ions as shown in Figure 1. Motivated by the 'effective' ternary phase diagram schemes used by Svensson, et al. ${ }^{31,91}$ we consider only charge-neutral complexes, where all charged components are paired with another component of opposite charge. The components include polymer-micelle complexes (with a volume fraction $\phi_{p m}$ ), polymer-salt complexes $\left(\phi_{p s}\right)$, and the solvent $\phi_{w}$. We note that, in this model, salt only exists as part of the polymer-salt complex, and thus we only consider the small-molecule anion that is the counterion to the polycation. There are no small-molecule cations included in this approach, a decision that drastically simplifies both the phase diagram and the model, and could be relaxed in future work. We note that it is possible to recover the volume fractions of the polymer and micelle components separately:

$$
\begin{array}{r}
\phi_{p m}=2\left\langle\phi_{p}\left(\Psi_{s}=M\right)\right\rangle+\phi_{m}^{\text {interior }} \\
\phi_{p s}=2\left\langle\phi_{p}\left(\Psi_{s}=S\right)\right\rangle \\
\left\langle\phi_{m}^{s s}\right\rangle=\left\langle\phi_{p}\left(\Psi_{s}=M\right)\right\rangle \\
\left\langle\phi_{s}\right\rangle=\left\langle\phi_{p}\left(\Psi_{s}=S\right)\right\rangle
\end{array}
$$

where $\phi_{m}^{\text {interior }}$ is the volume fraction of the surfactant-micelle that does not include the interacting surface sites, $\phi_{m}^{s s}$ is the volume fraction of the interacting micelle surface sites, $\left\langle\phi_{p}\left(\Psi_{s}=M\right)\right\rangle$ is the solution-averaged polymer concentration for the micelle-bound monomers. We assume the salt and micelle surface-stes bind to the monomers in a $1: 1$ volumetric ratio, and that $\left\langle\phi_{p}\right\rangle=\left\langle\phi_{p}\left(\Psi_{s}=M\right)\right\rangle+\left\langle\phi_{p}\left(\Psi_{s}=S\right)\right\rangle$

For a given set of concentrations $\phi_{p m}, \phi_{p s}$, and $\phi_{w}$, we seek a free energy expression that accounts for the microscopic interactions between the polymeric and micellar species. We consider an extension of the transfer matrix approach used in our prior work, ${ }^{37,73,87-90,99}$ which accounts for the ion pairing in a polyelectrolyte-polyelectrolyte coacervation to reflect 
the strong electrostatic interactions between the species. In this model, we similarly consider a constraint that all charged species are paired, such that each polyelectrolyte segment is considered to be paired with either a micelle surface site, or an oppositely-charged salt ion. This pairing requirement is the primary way in which electrostatics is included in our model, with long-range Coulomb interactions neglected due to the screening effect of the high concentration of charged species in coacervates. Thus the chain is treated as sequence of sites that are in one of two adsorption states, $\Psi_{s}=M$ for micelle-adsorbed segments, and $\Psi_{s}=S$ for salt-adsorbed segments. Each segments contributes a state-dependent adsorption energy $\left(\tilde{\varepsilon}_{m}, \tilde{\varepsilon}_{s}\right)$ to the potential energy of the chain, with an additional modification to $\tilde{\varepsilon}_{m}$ if the monomer is between two adjacent micelle surfaces; this is the second way in which electrostatics is included in our model, and is a phenomenological modification that accounts for complicated charge correlation effects. ${ }^{100,101}$ Our approach, elaborated upon below, considers all possible combinations of adsorption states by incorporating the two states into the calculation of the single-chain partition function in a self-consistent field theory (SCFT) calculation.

SCFT is used to model the conformation of the polyelectrolyte chains, as governed by the inhomogeneous distribution of micelle surface sites and the exclusion of polyelectrolyte and salt from the interior of the micelle structure. This micelle structure will be determined by performing straightforward Monte Carlo (MC) calculations on semiflexible bead-rod chains, which will represent a disordered wormlike micelle structure. Multiple samples of these simulation configurations will be mapped to the field in an SCFT calculation, by gridding space and designating each grid as a micelle surface site, micelle interior site, or an unoccupied site (i.e. micelle exterior).

The eventual free energy will consist of the free energy from this hybrid MC/SCFT calculation, as well as terms related to (1) the mixing entropy of the polymer and salt components, and (2) the excluded volume of the polymer and micellar components. We will consider each portion of the calculation in detail below. 


\section{Surfactant-Micelle Density Field}

The SCFT portion of our calculation will rely on having a potential field that accounts for the spatial distribution of the interacting surfactant-micelle surface sites, as well as the distribution of the surfactant-micelle interiors which exclude polymer and salt. The surfacesite, and interior-site distribution is predetermined via simulation snapshots of surfactantmicelle solutions at various surfactant-micelle densities (Figure 2a). We perform these MC simulations using a minimalist model of wormlike micelle structure, described using the potential $\beta U_{m}$ :

$$
\beta U_{m}=\beta U_{h s}+\beta U_{b}+\beta U_{\theta}=\sum_{i} \sum_{j>i} \beta u_{h s, i j}+\sum_{i}^{\prime} \beta u_{b, i, i+1}+\sum_{i}^{\prime} \beta u_{\theta, i, i+1, i+2}
$$

This potential is written as sums of pairwise contributions, with apostrophes on summations indicating that these potentials are only applied to beads within the same chains, of length $N=50$. The first term consists of hard-sphere potential contributions $\beta u_{h s, i j}$ between beads $i$ and $j$, that prevents overlap of micelle beads within a bead diameter $\sigma_{h s}=2.0$ :

$$
\beta u_{h s, i j}= \begin{cases}0 & r_{i j} \geq \sigma_{h s} \\ \infty & r_{i j}<\sigma_{h s}\end{cases}
$$

Here, $r_{i j}$ is the distance between beads $i$ and $j$. There is also a pairwise square well bonding potential $\beta u_{b, i, i+1}$, contributing to an overall bonding potential $\beta U_{b}$ connecting them into chain structures:

$$
\beta u_{b, i, i+1}= \begin{cases}0 & r_{i, i+1} \leq 1.01 \sigma_{h s} \\ \infty & r_{i, i+1}>1.01 \sigma_{h s}\end{cases}
$$

We finally include a harmonic bond angle potentials $\beta u_{\theta, i, i+1, i+2}$ that contribute to the overall bending potential $\beta U_{\text {theta }}$ :

$$
\beta u_{\theta, i, i+1, i+2}=\frac{\kappa_{\theta}}{2} \theta_{i, i+1, i+2}^{2}
$$


a)

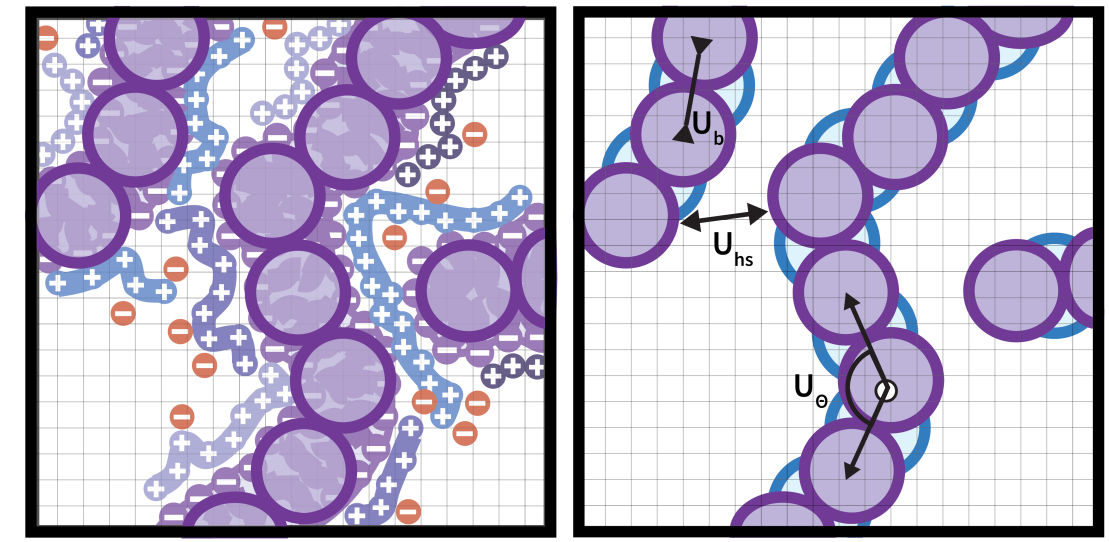

b)
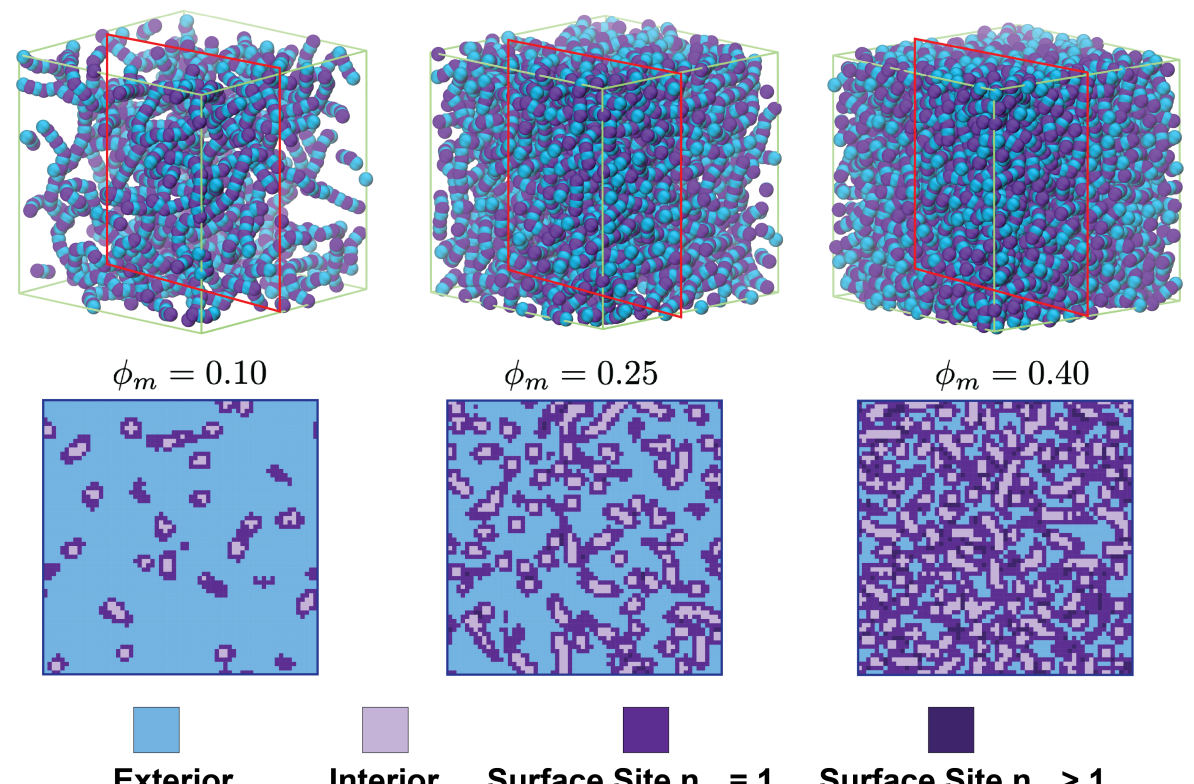

Interior Surface Site $n_{s s}=1$ Surface Site $n_{s s}>1$

Figure 2: (a, left) We will represent the work-like surfactant micelles in our model as a chain of beads (purple), where the bead diameter corresponds to the diameter of the surfactant micelle. (a, right) We will run MC simulations on these surfactant micelle beads, incorporating non-interacting interstitial beads (blue) in between the bonded beads (purple) to approximate the cylindrical shape of the micelles. The beads on the chain are connected by a bonded potential $U_{b}$, and they interact with the other interacting beads through a hard sphere potential $U_{h s}$. A bond-angle potential $U_{\Theta}$ describes the potential tied to the configuration of three consecutive bonded beads. (b) Representative simulation snapshots showing the interacting particles (purple) and the non-interacting interstitial particles (blue). The colormaps below correspond to a slice of the simulation snapshot. The blue areas of the slice correspond to the micelle exterior, the light purple areas correspond to the micelle interiors, and the darker shades of purple correspond to the surface sites, where darker shades represent points with multiple micelle surface sites. 
Here, we use a prefactor $\kappa_{\theta}=3.3$ that yields a persistence length consistent with typical values in the literature. ${ }^{102}$ The angle $\theta_{i, i+1, i+2}$ is defined between three subsequent beads, $i, i+1$, and $i+2$. The overall MC simulations were performed using the standard Metropolis criteria, where translational moves were accepted with a probability given by $p_{a c c}=\min \left[1, \exp -\Delta \beta U_{m}\right]$ where $\Delta \beta U_{m}$ is the change in the micelle potential for a given move.

These MC simulations were ran at hard sphere volume fractions from $\phi_{h s}=0.01$ to $\phi_{h s}=0.50$, with $\phi_{h s}=n_{h s} \frac{\pi \sigma_{h s}^{3}}{6} / L^{3}$, where $n_{h s}$ is the number of hard spheres and $L$ is the box length. The box length is set to $L=17 \sigma_{h s}$. Simulations were run for $100 \times 10^{6} \mathrm{MC}$ steps, which is sufficiently long to obtain equilibrium configurations.

Three sample configurations were selected from each of five equilibrated trajectories for every sampled value of $\phi_{h s}$, from which we extract the surface-site and interior site distribution. To do this, we first add non-interacting spheres between bonded hard-spheres to approximate a cylindrical micelle from the hard-sphere chains. The simulation box was discretized into $M^{3}=64^{3}$ collocation points, and each point was evaluated to determine if it resides on the surface of the micelle, the micelle interior, or micelle exterior (Figure 2b). Additionally, collocation points on the micelle surface were evaluated to determine the number of non-consecutive micelle segments, $n_{m, i}$ that were contained within the boundaries of the collocation point. Two potential fields are then generated from the discretization of the MC simulation, a micelle-interior energetic penalty field $\tilde{\Omega}(\mathbf{r})$, and the adsorption potential field corresponding to micelle-surface adsorbed segments $\tilde{\varepsilon}_{m}(\mathbf{r})$. Here we write potentials with a tilde to denote normalization by the thermal energy $k_{B} T$. The first potential field is an energetic penalty for placing the polyelectrolyte segment within the interior of the micelle, this field is set to $\tilde{\Omega}(\mathbf{r})=10$ for the micelle-interior sites, and $\tilde{\Omega}(\mathbf{r})=0$ elsewhere. The second potential field contains the contribution to the chain potential for a segment being in the micelle-adsorbed state, and this potential is set to $\tilde{\varepsilon}_{m}(\mathbf{r}) \rightarrow \infty$ for non-surface sites, $\tilde{\varepsilon}_{m}(\mathbf{r})=\tilde{\varepsilon}_{m, 0}$ for surface sites where $n_{m, i}=1$, and $\tilde{\varepsilon}_{m}(\mathbf{r})=2 \tilde{\varepsilon}_{m, 0}$ for 
surface sites where $n_{m, i}>1$. The differentiation between surface sites with different values of $n_{m, i}$ accounts for the possibility that two nearby charged micelle surfaces can induce strong polyelectrolyte-mediated correlation effects, such that there is an energetic benefit for nearby pairs of surfaces. ${ }^{100,101}$ The binding energy for salt-bound monomers is given by, $\tilde{\varepsilon}_{s}=\ln \left(A_{0} \phi_{s}\right)$, where $A_{0}=20.0$ as used in our previous work. ${ }^{73}$ Finally, the solution averaged micelle-adsorbed polymer segment density $\phi_{p, m}$, which corresponds to the solution averaged micelle surface-site density, can be calculated as follows:

$$
\left\langle\phi_{p, m}\right\rangle=\sum_{i} n_{m, i} \phi_{s s}
$$

where $\phi_{s s}$ is the local density of a surfactant micelle surface site. The potential fields determined from the MC simulations are used to inform the SCFT, where the partition function of a polyelectrolyte in an environment corresponding to that from the MC simulation can be evaluated.

\section{SCFT Model of Complexing Polyelectrolytes}

Self-consistent field theory (SCFT) will be used to consider the interactions of polyelectrolytes with surfactant micelles, and determine the conformational properties of these chains due to the potential fields determined from the MC simulations. The advantage to using SCFT is that it is able to account for the chain conformations and non-bonded interactions in the system, by decoupling the interparticle interactions and considering the system as a polymer density field that interacts with applied and internally-generated fields related in this case to the micelle structure. ${ }^{103}$ The internally-generated field will enforce the constraint of ion binding, and set a target monomer density that was determined via the MC simulations described in the previous section. Ultimately, this allows us to evaluate the partition function of the chains, and correspondingly the contributions to the free energy of the polyelectrolyte chain. 
We model the polyelectrolyte as a Gaussian chain, where the bond length between polymer segments form a Gaussian distribution. ${ }^{104}$ The bonding potential of the Gaussian chain can be expressed in the standard way: ${ }^{103,104}$

$$
\beta U_{b}[\mathbf{r}]=\frac{3}{2 b^{2}} \sum_{i}^{n_{p}} \int_{0}^{N_{s}} d s_{i}\left|\frac{d \mathbf{r}_{i}\left(s_{i}\right)}{d s_{i}}\right|^{2}
$$

where $b$ is the the segment length, $N_{s}$ is the number of segments in one chain, $n_{p}$ is the number of chains in the system, and $s$ corresponds to a coordinate along the chain contour. In addition to this bonded contribution to the system energy, segments along the chain can interact with the small molecule salt ions in the solution, as well as the oppositely-charged surfactant-micelle surface sites. This model considers the limit in which all the segments are paired with either a salt ion, or a micelle surface-site, and each segment is assigned a state $\Psi_{s}$ that corresponds to its paired charge $\left(\Psi_{s}=S\right.$ and $\Psi_{s}=M$ respectively). As such, the chain can be treated as a series of adsorption sites that contribute and adsorption energy corresponding to its state, $\tilde{\varepsilon}_{s}$ and $\tilde{\varepsilon}_{m}(\mathbf{r})$. Additionally, there is an energetic penalty for a polymer segment to occupy a space within the surfactant-micelle interior. This energetic penalty has been described in the previous section and is denoted as $\tilde{\Omega}(\mathbf{r})$. The contribution to the internal energy of the chain from the interactions with the opposite charges as well as the excluded volume of the micelle interior can be expressed as:

$$
\beta U_{n b}=\sum_{i}^{n_{p}} \int_{0}^{N_{s}} d s_{i}\left[\tilde{\varepsilon}_{m}\left(\mathbf{r}\left(s_{i}\right)\right) \delta\left(\Psi_{s_{i}}=M\right)+\tilde{\varepsilon}_{s} \delta\left(\Psi_{s_{i}}=S\right)+\Omega\left(\mathbf{r}\left(s_{i}\right)\right)\right]
$$

Here we have used $\delta\left(\Psi_{s_{i}}=X\right)$ as a way to denote the specific state $\Psi$ of monomer $s_{i}$, two options $X=S, M$ that must be considered at each grid point in enumerating the single-chain partition function that will be discussed in detail later. 
From these potentials, the configurational partition function can be represented as:

$$
\mathcal{Z}_{c}=\frac{z_{0}^{n_{p}}}{n_{p} !} \prod_{i}^{n_{p}} \sum_{\left\{\Psi_{s_{i}}\right\}} \int \mathcal{D} \mathbf{r}_{i} \exp \left(-\beta\left(U_{b}\left[\mathbf{r}^{n_{p} N_{s}}\right]+U_{n b}\left[\mathbf{r}^{n_{p} N_{s}}\right]\right)\right)
$$

This particle-based partition function is transformed into a field-based partition function using the particle to field transformation described in Fredrickson's monograph. ${ }^{103}$ To carry out this procedure, we first define the microscopic polymer segment density operator, which corresponds to the micelle-adsorbed polymer segments, as the following:

$$
\hat{\rho}_{p, m}(\mathbf{r})=\sum_{i}^{n_{p}} \int d s_{i} \delta\left(\mathbf{r}-\mathbf{r}\left(s_{i}\right)\right) \delta\left(\Psi_{s_{i}}=M\right)
$$

The reason to define this quantity, which reports only the micelle-bound monomers, is to enforce the constraint that these monomers have the same density as the micelle surface site density field $\rho_{m}(\mathbf{r})$ that was determined from the MC simulation and gridding procedure described earlier so that $\rho_{m}(\mathbf{r})=\hat{\rho}_{p, m}$ for all $\mathbf{r}$. We consider a delta functional constraint:

$$
\delta\left[\rho_{m}-\hat{\rho}_{p, m}\right]=\int \mathcal{D} \omega_{c} \exp \left(i \int d \mathbf{r} \omega_{c}(\mathbf{r})\left[\rho_{m}(\mathbf{r})-\hat{\rho}_{p, m}(\mathbf{r})\right]\right)
$$

Here we have written the delta functional as a functional integral over an auxiliary constraining field $\omega_{c}$, an expression that can be directly incorporated into the partition function:

$$
\begin{aligned}
& \mathcal{Z}_{c}=\frac{z_{0}^{n_{p}}}{n_{p} !} \int \mathcal{D} \rho_{m} \int \mathcal{D} \omega_{c} \prod_{i}^{n_{p}} \int \mathcal{D} \mathbf{r}_{i} \sum_{\Psi_{i}} \exp \left(-\beta U_{b}+i \int d \mathbf{r} \omega_{c}\left(\rho_{m}-\hat{\rho}_{p, m}\right)\right) \\
& \exp \left(-\sum_{i}^{n_{p}} \int_{0}^{N_{s}} d s_{i}\left[\tilde{\varepsilon}_{m}\left(\mathbf{r}\left(s_{i}\right)\right) \delta\left(\Psi_{s_{i}}=M\right)+\tilde{\varepsilon}_{s} \delta\left(\Psi_{s_{i}}=S\right)+\Omega\left(\mathbf{r}\left(s_{i}\right)\right)\right]\right)
\end{aligned}
$$

We combine all of the chain-specific terms in the exponential term of the partition function into a single-chain partition function $Q\left[i \omega_{c}\right]$, given by: 


$$
\begin{aligned}
& Q\left[i \omega_{c}\right]=\frac{1}{Z_{0}} \int \mathcal{D} \mathbf{r}_{i} \sum_{\Psi_{i}} e^{-\beta U_{b}} \times \\
& \times \exp \left(-\int_{0}^{N_{s}} d s_{i}\left[\left\{\tilde{\varepsilon}_{m}\left(\mathbf{r}\left(s_{i}\right)\right)+i \omega_{c}\left(\mathbf{r}\left(s_{i}\right)\right)\right\} \delta\left(\Psi_{s_{i}}=M\right)+\tilde{\varepsilon}_{s} \delta\left(\Psi_{s_{i}}=S\right)+\Omega\left(\mathbf{r}\left(s_{i}\right)\right)\right]\right)
\end{aligned}
$$

The factor $Z_{0}$ is the path integral of a free polymer in zero field; this quantity is independent of the density and auxiliary fields, is extensive, and depends on V. It can therefore be expressed as $Z_{0}=g_{N} V$ where $g_{N}$ is a factor that is thermodynamically unimportant. We have used the identity $-i \int d \mathbf{r} \omega_{c} \hat{\rho}_{p, m}=-i \sum_{i}^{n_{p}} \int_{0}^{N_{s}} d s_{i} \omega_{c}\left(\mathbf{r}\left(s_{i}\right)\right) \delta\left(\Psi_{s_{i}}=M\right)$ to include the constraining field directly into the exponential of the single-chain partition function. We can thus write the partition function as:

$\mathcal{Z}_{c}=\frac{z_{0}^{n_{p}}}{n_{p} !} \int \mathcal{D} \rho_{m} \int \mathcal{D} \omega_{c} \exp \left(i \int d \mathbf{r} \omega_{c} \rho_{m}\right)\left[Z_{0} Q\left[i \omega_{c}\right]\right]^{n_{p}}=\frac{\left(z_{0} g_{N} V\right)^{n_{p}}}{n_{p} !} \int \mathcal{D} \rho_{m} \int \mathcal{D} \omega_{c} e^{-\beta \mathcal{H}\left[\rho_{m}, \omega_{c}\right]}$

Here we have defined a Hamiltonian:

$$
\mathcal{H}\left[\rho_{m}, \omega_{c}\right]=-i \int d \mathbf{r} \omega_{c}(\mathbf{r}) \rho_{m}(\mathbf{r})-n_{p} \ln Q\left[i \omega_{c}\right]
$$

In this model, the micelle surface sites are fixed for a given MC-determined micelle configuration, therefore the functional integral over $\rho_{m}$ has only one term and can be left out of the SCFT calculation; in effect, we approximate an integral over this variable by performing this calculation over multiple instances of these micelle configurations for a given micelle and polymer concentration. To solve for the partition function, we invoke a meanfield assumption that there is a single field $\omega_{c}^{*}$ that minimizes the Hamiltionian $\mathcal{H}\left[\omega_{c}^{*}\right]$, and that this field dominates the path integral over $\omega_{c}$ of the partition function. Therefore we 
can approximate $\mathcal{Z}_{c}$ as:

$$
\mathcal{Z}_{c} \approx \frac{\left(z_{0} g_{N} V\right)^{n_{p}}}{n_{p} !} \exp \left(-\mathcal{H}\left[\omega_{c}^{*}\right]\right)
$$

This allows direct calculation of the mean-field free energy $\mathcal{F}_{S C F T}=k_{B} T \ln \mathcal{Z}_{c}$ for the polyelectrolyte portion of the calculation, but first requires determination of the extremized Hamiltonian $\mathcal{H}\left[\omega_{c}^{*}\right]$ via a numerical SCFT calculation.

\section{Numerical solution to SCFT}

The practical determination of the extremized Hamiltonian $\mathcal{H}\left[\omega_{c}^{*}\right]$ follows the standard SCFT approach. This requires numerical determination of the single chain partition function via calculation of the single chain propagator in the constraining field $i \omega_{c}(\mathbf{r})$, in conjunction with calculating the density field of micelle-bound monomers $\hat{\rho}_{p, m}$ that is used to determine the magnitude of the same constraining field.

The propagator function $q\left(\mathbf{r}, s, \Psi_{s} ;\left[i \omega_{c}\right]\right)$ accounts for the Boltzmann weight of a polymer segment from the start of the chain until a defined index $s$, state $\Psi_{s}$, and location $\mathbf{r}$ in a field $i \omega_{c}(\mathbf{r})$. This allows for a recursive evaluation for the partition function of the whole chain via a Markov process, with the propagator is defined as:

$$
q\left(\mathbf{r}, s+\Delta s, \Psi_{s} ;\left[i \omega_{c}\right]\right)=\int d \mathbf{r}^{\prime} \sum_{\Psi_{s}^{\prime}} \Phi\left(\mathbf{r}, \Psi_{s} \mid \mathbf{r}^{\prime}, \Psi_{s^{\prime}}\right) q\left(\mathbf{r}^{\prime}, s, \Psi_{s}^{\prime},\left[i \omega_{c}\right]\right)
$$

where $\Phi\left(\mathbf{r}, \Psi_{s} \mid \mathbf{r}^{\prime}, \Psi_{s^{\prime}}\right)$ is the transition weight for a displacement from $\mathbf{r}$ to $\mathbf{r}^{\prime}$ for the chain segment spanning $s$ to $s^{\prime}=s+\Delta s$, where $s$ and $s^{\prime}$ are in states $\Psi_{s}$ and $\Psi_{s^{\prime}}$ respectively. This transition probability density can be separated into a diffusive component ( $\mathbf{r}$ dependent, $\Phi_{\mathbf{r}}$ ) and a potential field component ( $\Psi_{s}$ dependent, $\Phi_{\Psi}$ ):

$$
q\left(\mathbf{r}, s+\Delta s, \Psi_{s},\left[i \omega_{c}\right]\right)=\sum_{\Psi_{s^{\prime}}} \Phi_{\Psi}\left(\Psi_{s} \mid \Psi_{s^{\prime}}\right) \int d \mathbf{r}^{\prime} \Phi_{\mathbf{r}}\left(\mathbf{r} \mid \mathbf{r}^{\prime}\right) q\left(\mathbf{r}^{\prime}, s, \Psi_{s^{\prime}},\left[i \omega_{c}\right]\right)
$$


We can numerically evaluate this Chapman-Kolmogorov expression through a two-step procedure; first, the integral $\int d \mathbf{r}^{\prime} \Phi_{\mathbf{r}}\left(\mathbf{r} \mid \mathbf{r}^{\prime}\right) q\left(\mathbf{r}^{\prime}, s, \Psi_{s}^{\prime},\left[i \omega_{c}\right]\right)$ can be calculated by numerically integrating the diffusive portion of the propagator for a Gaussian chain over a segment of length $\Delta s:{ }^{103}$

$$
\frac{\partial}{\partial s} q\left(\mathbf{r}, s, \Psi_{s}^{\prime} ;\left[i \omega_{c}\right]\right)=\frac{b^{2}}{6} \nabla^{2} q\left(\mathbf{r}, s, \Psi_{s}^{\prime} ;\left[i \omega_{c}\right]\right)
$$

We refer readers to the monograph by Fredrickson ${ }^{103}$ for methods to perform this standard numerical calculation; we use a pseudo-spectral method to evaluate this portion of the propagator. The second step is to evaluate the energetic contribution, which enters via the matrix $\Phi_{\Psi}\left(\Psi_{s} \mid \Psi_{s^{\prime}}\right)$ of Boltzmann factors related to two adjacent adsorption states $\Psi_{s}$ and $\Psi_{s^{\prime}}$. While in principle this matrix could account for interactions between adjacent monomers, as is done in our prior work, ${ }^{37,73,87-90,99}$ we choose a simple non-interacting form for this matrix:

$$
\Phi_{\Psi}\left(\Psi_{s} \mid \Psi_{s^{\prime}}\right)= \begin{cases}e^{-\left(\tilde{\varepsilon}_{s}+\Omega(\mathbf{r})\right)} & \Psi_{s}=S \\ e^{-\left(i \omega_{c}(\mathbf{r})+\tilde{\varepsilon}_{m}+\Omega(\mathbf{r})\right)} & \Psi_{s}=M\end{cases}
$$

These two steps can be used to integrate from an initial condition $q\left(\mathbf{r}, s=0, \Psi_{s} ;\left[i \omega_{c}\right]\right)=1$, to obtain the single chain partition function $Q$ that is given by:

$$
Q\left[i \omega_{c}\right]=V^{-1} \sum_{\Psi_{N}} \int d \mathbf{r} q\left(\mathbf{r}, s=N, \Psi_{N} ;\left[i \omega_{c}\right]\right)
$$

Solving for the propagator along the chain is the most computationally costly calculation of our model, as the MC simulations of the surfactant-micelle solution was discretized into $M^{3}=64^{3}$ collocation points.

Calculation of the propagator allows for the determination of the micelle density field from the operator $\hat{\rho}_{p, m}$ :

$$
\hat{\rho}_{p, m}(\mathbf{r})=-n_{p} \frac{\delta \ln Q\left[i \omega_{c}\right]}{i \omega_{c}(\mathbf{r})}=\frac{\left\langle\phi_{p}\right\rangle}{N_{p}} \frac{\int_{s=0}^{s=N_{s}} d s q\left(\mathbf{r}, s, M,\left[i \omega_{c}\right]\right) q\left(\mathbf{r}, N_{s}-s, M,\left[i \omega_{c}\right]\right)}{1 / N_{g} \sum_{\Psi} q\left(\mathbf{r}, N, \Psi,\left[i \omega_{c}\right]\right)}
$$


The extremization of the Hamiltonian is performed by satisfying the constraint that $\rho_{m}(\mathbf{r})=$ $\hat{\rho}_{p, m}(\mathbf{r})$ for all points $\mathbf{r}$, by modifying the auxiliary field $i \omega_{c}(\mathbf{r})$. Starting with an initial guess for $i \omega_{c}(\mathbf{r})$, we evaluate the propagator via Equation 21 to determine single chain partition function in Equation 24. This can be used to calculate the density operator via Equation 25. Comparison of this density operator, which represents the density of micelle-bound monomers, with the density of micelle surface sites informs the update of the constraining field:

$$
i \omega_{c}(\mathbf{r})=i \omega_{c}(\mathbf{r})^{\prime}-\alpha \frac{\delta H[\omega]}{i \delta \omega_{c}(\mathbf{r})}=i \omega_{c}(\mathbf{r})^{\prime}-\alpha\left(\rho_{m}(\mathbf{r})-\hat{\rho}_{p, m}(\mathbf{r})\right)
$$

Here, the parameter $\alpha$ controls the rate at which the difference of the two density fields converge by setting the per-iteration correction to the constraining field, which is chosen to converge as quick as possible while still maintaining numerical stability.

\section{Solution free energy}

The iterative SCFT calculation obtains an auxiliary constraining field $i \omega_{c}^{*}(\mathbf{r})$ that extremizes $\mathcal{H}$, allowing us to define a free energy from Equation 19 via the relationship:

$$
\beta \mathcal{F}_{S C F T} / V=\frac{1}{V} \ln \mathcal{Z}_{c}=-\frac{n_{p}}{V} \ln \left(z_{0} g_{N} V\right)+\frac{n_{p}}{V} \ln n_{P}-\mathcal{H}\left[i \omega_{c}^{*}(\mathbf{r})\right]
$$

Assuming a reference volume $\nu_{p}$ for the monomer, we can write the corresponding free energy in terms of the numerical gridded system (i.e. over the $M^{3}$ collocation points):

$$
\frac{\nu_{p} \beta F_{S C F T}}{V}\left(\phi_{p m}, \phi_{p s}\right)=\frac{\nu_{p} \beta}{V} F_{0}+\frac{\phi_{p}}{N_{p}} \ln \phi_{p}-i \frac{1}{M^{3}} \sum_{i}^{M^{3}} \omega_{c, i} \phi_{p_{m}, i}-\frac{\phi_{p}}{N_{p}} \ln Q\left[i \omega_{c}\right]
$$

Here, $\phi_{p_{m}, i}$ corresponds to the micelle adsorbed monomer volume fractions, and we use a subscript for $\omega_{c, i}$ and $\phi_{p_{m}, i}$ to denote that these are quantities located at discrete grid points $i$. We also include an arbitrary reference free energy $F_{0}$ that is thermodynamically irrelevant 
but comes from both other constants in the partition function and the conversion to volume fractions $\phi_{p}$. Again, $\phi_{p m}$ and $\phi_{p s}$ are the polymer-micelle and polymer-salt complex volume fractions, which can be directly related to the actual values used in the expression $\left(\phi_{p}, \phi_{s}\right.$, $\left.\phi_{m}\right)$ due to electroneutrality.

This result from the SCFT calculation only includes the contribution due to the free energy of the polymeric species, particularly (1) their interactions with surrounding species, (2) their conformational degrees of freedom, and (3) their translational entropy. We also include a number of other terms to account for other interactions not included in this calculation:

- We must include the salt translational entropy, which is the standard expression $\nu_{p} \beta F_{s, \text { trans }} / V=\phi_{s} \ln \phi_{s}$.

- In connection with our previous work on polyelectrolyte-polyelectrolyte coacervation theories, ${ }^{73}$ we include a phenomenological cubic term to account for excluded volume of the polymer-salt complex:

$$
\frac{\nu_{p} \beta F_{p s, e v}}{V}=\xi\left[\frac{\phi_{p s}}{\left(1.0-\left\langle\phi_{p, m}\right\rangle-\phi_{m}^{\text {interior }}\right)}\right]^{3}
$$

We note that the denominator reflects the occupation of volume by the micellar components, which contributes to an increase in the effective concentration of the polymeric species. We choose $\xi=20.0$, which is similar to the value used in our previous work. ${ }^{37,73,87,89,90}$

- We approximate the excluded volume contribution from the surfactant-micelle chains using the Carnahan Starling equation of state, ${ }^{105}$

$$
\frac{\nu_{p} \beta F_{c s}}{V}\left(\phi_{m}^{\prime}\right)=\left(\frac{\nu_{m}}{\nu_{p}}\right)\left(\frac{\phi_{m}^{\prime}}{\nu_{m}}\right) \frac{4 \phi_{m}^{\prime}-3 \phi_{m}^{\prime 2}}{\left(1-\phi_{m}^{\prime}\right)^{2}}
$$

where $\nu_{m}$ is the volume of the micelle hard sphere particle from the MC simulations, and $\phi_{m}^{\prime}$ is the volume fraction of the micelle chain including the non-interacting interstitial 
particles and the hard-sphere chain particles from the MC simulations.

This leads to an overall expression for the free energy of the system, $\mathcal{F}$ :

$$
\begin{array}{r}
\frac{\nu_{p} \beta \mathcal{F}}{V}=\frac{\nu_{p} \beta}{V}\left(F_{S C F T}+F_{s, \text { trans }}+F_{p s, e v}+\beta F_{c s}\right)= \\
=\frac{\nu_{p} \beta}{V} F_{0}+\frac{\phi_{p}}{N_{p}} \ln \phi_{p}+\phi_{s} \ln \phi_{s}-i \frac{1}{M^{3}} \sum_{i}^{M^{3}} \omega_{c, i} \phi_{p_{m}, i}-\frac{\phi_{p}}{N_{p}} \ln Q\left[i \omega_{c}\right]+ \\
+\xi\left[\frac{\phi_{p s}}{\left(1.0-\left\langle\phi_{p, m}\right\rangle-\phi_{m}^{\text {interior }}\right)}\right]^{3}+\left(\frac{\nu_{m}}{\nu_{p}}\right)\left(\frac{\phi_{m}^{\prime}}{\nu_{m}}\right) \frac{4 \phi_{m}^{\prime}-3 \phi_{m}^{\prime 2}}{\left(1-\phi_{m}^{\prime}\right)^{2}}
\end{array}
$$

This free energy expression will be used to map out the phase behavior of coacervation in polyelectrolyte-micelle systems, and we note that it implicitly includes both the MC simulation results and SCFT calculations in the determination of the constraining field $\omega_{c}$ and the single-chain partition function $Q$. These thus include key assumptions that will limit the scope of our predictions; first, while we take an ensemble of MC simulations as the foundation for the SCFT, we are limited by the numerical averaging over stochasticallydetermined micelle structures. Importantly, these structures are also not themselves affected by the polyelectrolyte interactions, which is a major assumption that we make in our work. We justify this lack of self-consistency by noting that the dense branch of the coacervate phase separation is almost always highly packed (ca. $\phi_{m} \approx 0.5$ ) and the polyelectrolyte-induced attraction would likely only perturb this structure slightly. Indeed, strong perturbations would likely induce ordering in the micellar structures, a situation we explicitly neglect in this simplified theory. Even in the dilute branch of the coacervate phase separation (i.e. the supernatant) will have a relatively high concentration of micelles (ca. $\phi_{m} \approx 0.1-0.3$ ), so again we a posteriori justify our assumption that this lack of self-consistency is not a major deficiency of this model.

Another major assumption is in the nature of the polyelectrolyte interactions, which are treated as pairwise 'ion binding' events that come with binding energies $\tilde{\varepsilon}_{m}$ and $\tilde{\varepsilon}_{s}$ that must be parameterized. We treat these as tunable parameters, and note (1) they can be ostensi- 
bly determined from simulation, as has been done in our prior work, ${ }^{37,73,87,106}$ where (2) this approach has been used to great effect in describing a number of experimentally-validated aspects of coacervation (e.g. sequence, ${ }^{72}$ branching, ${ }^{87,106}$ polyampholyte self-coacervation, ${ }^{73}$ and multivalent ions ${ }^{87}$ ). Finally, we note that we invoke the traditional mean-field approximation for the SCFT portion of the calculation. This may be relaxed with more advanced field theoretic calculations, ${ }^{103}$ but expect only quantitative improvements that would be small compared to the other approximations we are making. These approximations represent opportunities to further refine this model, which we will save for future efforts.

\section{Results}

\section{Free Energy Landscape}

The simulation informed, self-consistent field theory model developed in this paper allows us to determine the system free energy $\mathcal{F}$ for a wormlike surfactant-micelle and polyelectrolyte solution. Solution behavior of the system can be determined from the free energy manifold evaluated under a number of key system variables; we will focus on phase behavior as a function of the polymer-micelle complex concentration $\phi_{p m}$ and the polymer-salt complex concentrations $\phi_{p s}$, which are the main compositional variables in our model. There are also a number of molecular parameters that can be set within the model, and reflect the values that would be determined by the specific species considered. For example, we define the ratio of the ideal radius of gyration of the polymer and the micelle radius, $\Theta=R_{g} / R_{m}$ to quantify the difference in length scales between the MC simulations and the field theory. The dependence of $R_{g}=N_{p}^{1 / 2} b / \sqrt{6}$ on $\Theta$ links this parameter to the degree of polymerization of the polyelectrolyte, $N_{p}$, as well as the segment size of the polyelectrolyte, $b$. Another tunable molecular parameter in the model is the concentration of the surfactant-micelle surface site, $\phi_{s s}$. This parameter sets the volume of the surface site, and it is assumed that polyelectrolyte monomers and micelle surface-site interact with each other in a $1: 1$ 
volumetric ratio. Therefore the parameter $\phi_{s s}$ determines how much polymer interacts with each micelle surface site. Finally, we tune the strength of interaction between the oppositely charged polyelectrolyte and micelle surface sites via the binding energy for micelle-adsorbed monomers, $\tilde{\varepsilon}_{m, 0}$. This parameter accounts for both the bare binding energy between the two species, but also accounts for correlation-driven attractions between nearby surfaces that in our model are included via the factor of 2 when two or more micelles share a surface site. While the exact nature of this correlation could be refined with molecular simulation ${ }^{100}$ or field theoretic arguments, ${ }^{101}$ our model keeps this at the level of a phenomenological parameter by virtue of the magnitude of $\tilde{\varepsilon}_{m, 0}$.
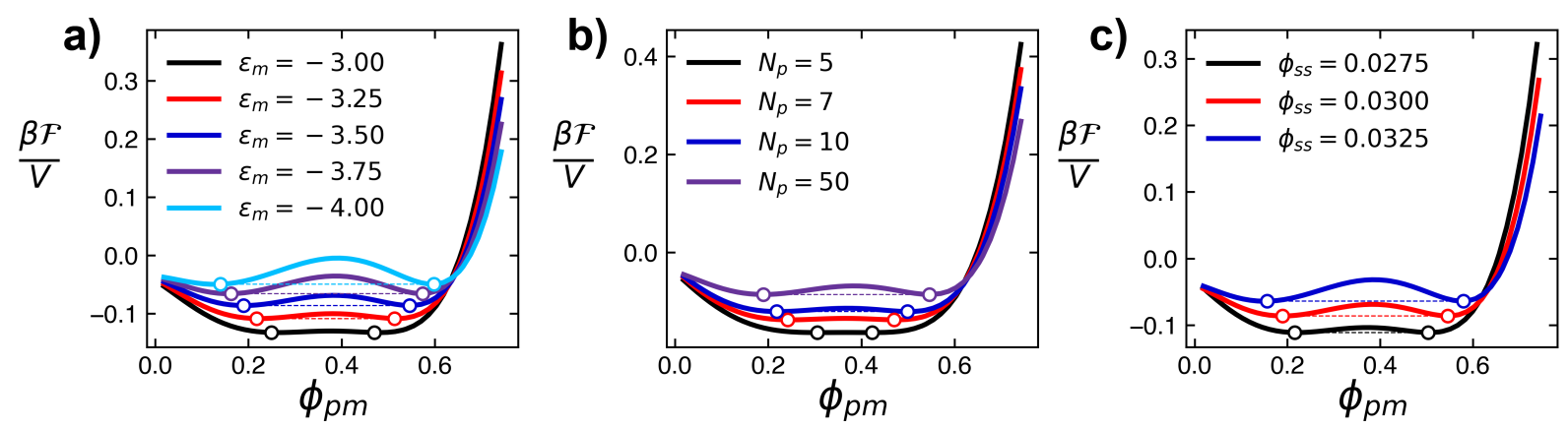

Figure 3: Plots of the free energy density vs. polymer-micelle complex concentration while varying (a) micelle binding energy $\tilde{\varepsilon}_{m}$, (b) degree of polymerization $N_{p}$, and (c) surface site volume fraction $\phi_{s s}$. A linear shift factor of $a \times \phi_{p m}$ is added to the free energy density, with prefactor $a$ chosen such that the common tangents along the curve have approximately equal values for the free energy density. The common tangents are connected by a dotted line.

In the limiting case where the solution-average polymer concentration is equal to the solution-average micelle surface site concentration, all of the monomers will be in the micelleadsorbed state, therefore $\phi_{p s}=0$. In this zero polymer-salt complex limit, the free energy can be expressed in terms of only the polymer-micelle complex concentration. The manifold in this limit will therefore be one-dimensional, and the binodal will be the common-tangent points $\phi_{p m}^{A}$ and $\phi_{p m}^{B}$, where $\left(\frac{d(\beta \mathcal{F} / V)}{d \phi_{p m}}\right)_{\phi_{p m}=\phi_{p m}^{A}}=\left(\frac{d(\beta \mathcal{F} / V)}{d \phi_{p m}}\right)_{\phi_{p m}=\phi_{p m}^{B}}$. The common-tangent points are the coexisting compositions in the two phase solution (with the phases denoted as $A$ and $B$ ). Figure 3 shows the system free energy as function of $\phi_{p m}$ with $\phi_{p s}=0$, while 
comparing the model parameters $N_{p}, \tilde{\varepsilon}_{m}$, and $\phi_{s s}$. In order to better visualize the common tangents of the free energy, a thermodynamically irrelevant term linear in polymer-micelle complex concentration was added to the free energy, $a \times \phi_{p m}$. Values for $a$ where chosen such that the free energy density at the two common tangent points are approximately equal so that it is easier to visualize the common tangents, shown in the circular markers. Figure 3A shows the free energy density curve for different values for the micelle-surface binding energy $\tilde{\varepsilon}_{m}$, with $\Theta=0.4, N_{p}=50$, and $\phi_{s s}=0.03$. The supernatant polymer-micelle complex concentration decreases with decreasing $\tilde{\varepsilon}_{m}$, and the coacervate polymer-micelle complex concentration increases with decreasing $\tilde{\varepsilon}_{m}$. Decreasing the magnitude of the micelle-surface binding energy leads to a shrinking of the coexistence concentrations where polymer-micelle complexes undergo macroscopic phase separation, illustrating that strong correlation interactions are necessary to observe the formation of polymer-micelle coacervates. Figure 3B shows the free energy density curves for different degree of polymerization for the polyelectrolyte $N_{p}$, and the other model parameters are set to $\tilde{\varepsilon}_{m}=-3.5$, and $\phi_{s s}=0.03$. For $N_{p}=50$, the ratio of the radius of gyration of the polyelectrolyte to the surfactant-micelle radius is set to $\Theta=0.4$. $\Theta$ is scaled for the other values of $N_{p}$ to maintain a consistent segment size, $\Theta^{\prime}=\Theta \sqrt{N_{p}^{\prime} / N_{p}}$. The change in $\beta \mathcal{F} / V$ versus $\phi_{p m}$ between the degree of polymerization values of $N_{p}=10$ to $N_{p}=50$ is small given the large change in chain length, with only minor changes to the common tangent locations that result in a slight increase in the coexistence region. Conversely, a decrease of the degree of polymerization from $N_{p}=10$ to $N_{p}=5$ significantly shifts the common tangent points, so that the window for phase separation becomes significantly diminished. In the low $N_{p}$ limit, the polymer translational entropy dominates the free energy and favors mixing, however as $N_{p}$ is increased this term becomes negligible. This is similar to what is seen to occur in polyelectrolyte-polyelectrolyte coacervates, which exhibit phase diagrams that become essentially insensitive to the degree of polymerization at sufficiently high chain lengths. ${ }^{42,107}$ Figure 3C shows the free energy density curves for different values for $\phi_{s s}$. The degree of polymerization is set to $N_{p}=50$, 
and the micelle-surface binding is set to $\tilde{\varepsilon}_{m}=-3.5$. As the surface site volume fraction $\phi_{s s}$ is increased, the amount of polymer each surface site can bind is increased, therefore the free energy stabilization as a result of attractive polyelectrolyte-micelle interaction is enhanced. This increase in polymer-micelle interactions can stabilize coacervate phases with higher macroion densities. As the surface site volume fraction is increased, the coacervate phase macroion densities in $3 \mathrm{C}$ are increased as indicated by the common-tangent points, the surfactant phase macroion volume fractions correspondingly are decreased with increasing surface site volume fraction.
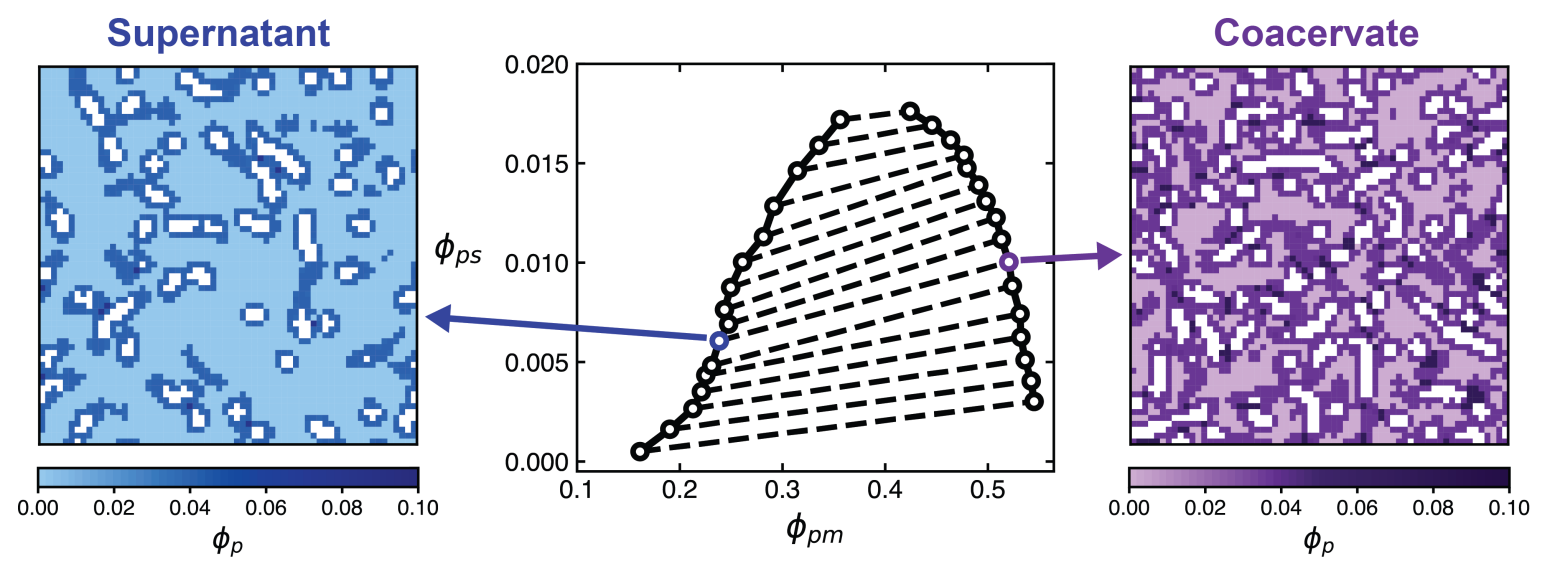

Figure 4: Phase Diagram (center) for a polyelectrolyte-surfactant system, in the polymersalt complex volume fraction $\phi_{p s}$ versus polymer-micelle complex volume fraction $\phi_{p m}$ plane. The phase diagram is flanked on each site by a density map of the polymer concentration $\phi_{p}$ (see legends) in a 2D slice of the simulation space for the indicated supernatant (blue) composition and corresponding coacervate (purple) composition. The phase diagram shows the binodal for the system with the degree of polymerization $N_{p}=50$, micelle binding energy set $\tilde{\varepsilon}_{m}=-3.5$, and surface site volume fraction $\phi_{s s}=0.03$. The tie lines connect the two compositions in phase coexistence.

In the case where the solution-average polymer concentration $\left\langle\phi_{p}\right\rangle$ is greater than the solution-average micelle surface-site density $\left\langle\phi_{s s}\right\rangle$, the micelle surface-sites will still be completely occupied by polymer while the remaining polymer will complex with oppositelycharged salt ions. The free energy density manifold will be a surface, and therefore the binodal will be represented by a curve in $\phi_{p s}$ versus $\phi_{p m}$ space. The binodal of the free energy manifold is numerically evaluated, and plotted in Figure 4 for $N_{p}=50, \tilde{\varepsilon}_{m}=-3.5$, and 
$\phi_{s s}=0.03$. The area below and between the coexistence curves is a two-phase coexistence area, while the area above and outside the coexistence curves is the single-phase region. Dashed tie lines connect the two coexisting phase concentrations, characterized by micelledilute (i.e. supernatant) and micelle-concentrated (i.e. coacervate) phases. Increasing the solution-average polymer-salt concentration $\phi_{p s}$ in a phase-separated solution monotonically decreases the difference between $\phi_{p m}$ in the coexisting phases, up to a critical polymer-salt complex concentration. Due to the use of MC simulations to determine representative micelle structures for the SCFT calculation, we can plot example polymer-micelle complex density fields as shown in Figure 4. This figure plots slices of the density fields for the coexisting supernatant and coacervate phases selected in purple and blue respectively. The polymer chains are localized onto the micelle surface and are excluded from the micelle interiors, and thus outline the wormlike micelle structure. Surface sites in contact with more than one surfactant micelle are able bind more polymer, and thus these sites are higher in polymermicelle complex concentration than the rest of the micelle surface sites. We can see from the density field slices that there are considerably more of these higher density sites in the coacervate phase than in the supernatant, which drives the formation of the micelle-dense phase; however, we note that these favorable interactions are necessarily dilute compared to a polyelectrolyte-polyelectrolyte coacervate, by virtue of the large size of the micelles. Furthermore, the large size of the micelles also prevents them from being partitioned exclusively to the coacervate due to significant excluded volume repulsions. This balance of dilute attractions and excluded volume repulsions is reflected in the relatively small difference in the $\phi_{p m}$ concentration between the phases, where both phases have a significant number of micelles. This contrasts with the analogous phase diagrams in polyelectrolyte-polyelectrolyte coacervates, where there are often orders of magnitude in difference between the polyelectrolyte concentrations in the supernatant versus the coacervate phases. ${ }^{42,43,49,107,108}$

Figure 5 compares the phase diagrams for different values for the model parameters $N_{p}$, $\tilde{\varepsilon}_{m}$, and $\phi_{s s}$. Figure $5 \mathrm{~A}$ shows the two phase coexistence curves for different values for the 

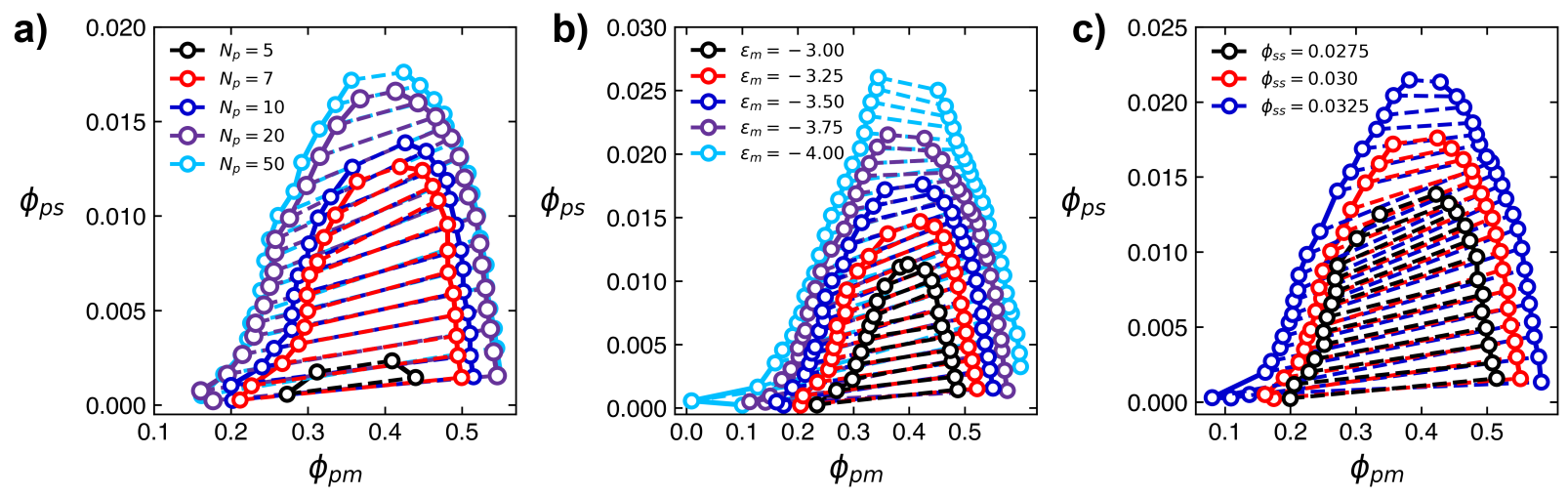

Figure 5: Plots comparing the $\phi_{p s}$ versus $\phi_{p m}$ phase diagrams for different values of (a) degree of polymerization $N_{p}$, (b) micelle binding energy $\tilde{\varepsilon}_{m}$, and (c) surface site volume fraction $\phi_{s s}$. The parameters not being varied in each plot are held constant to the following values: $N_{p}=50, \tilde{\varepsilon}_{m}=-3.5$, and $\phi_{s s}=0.03$.

degree of polymerization, from $N_{p}=5$ to $N_{p}=50$. The micelle binding energy is set to $\varepsilon=-3.5$, and, and the surface site concentration is set to $\phi_{s s}=0.03$. The minimum value for $N_{p}$ which shows phase separation is $N_{p}=5$. Lowering the degree of polymerization further increases the free energy density contribution from the polymer translational entropy which stabilizes the single phase solution. As $N_{p}$ is initially increased above $N_{p}=5$, the two-phase region expands significantly. However, above $N_{p} \approx 20$, there is very little change as the value of $N_{p}$ is further increased. This is again attributed to the polymer translational entropy become negligible compared to the other terms in the free energy denstiy.

Figure 5B shows the two-phase coexistence curves for differing values for the micelle binding energy $\tilde{\varepsilon}_{m}$, with a degree of polymerization of $N_{p}=50$, and the surface site concentration set to $\phi_{s s}=0.03$. As the magnitude of $\tilde{\varepsilon}_{m}$ becomes larger (i.e. becomes more negative), the coexistence region increases significantly due to the increased attraction between monomers and the surfactant-micelle surface sites. This increased propensity to phase separate is consistent with the idea that phase separation between oppositely charged surfactant-micelles and polyelectrolytes is driven by the electrostatic attraction between the two species; we note that no phase separation is observed if we remove the factor of 2 that multiplies $\tilde{\varepsilon}_{m, 0}$ and phenomenologically accounts for correlation-driven attraction between shared (i.e. $n_{m, i}=2$ ) 
surface sites.

Figure $5 \mathrm{C}$ shows the phase diagrams for three different values for the surface site volume fraction $\phi_{s s}$, with a degree of polymerization of $N_{p}=50$, and the micelle binding energy set to $\tilde{\varepsilon}_{m}=-3.5$. As $\phi_{s s}$ is increased, the two-phase coexistence region is increased, along with the coacervate phase polymer-micelle complex concentration. Similar to $\tilde{\varepsilon}_{m}$, this value tunes the interactions between the polymer and micelle. $\tilde{\varepsilon}_{m}$ changes the strength of attraction between the oppositely charged macroions, and $\phi_{s s}$ changes the amount of polymer that binds to a micelle surface site. Changes to $\phi_{s s}$ results in an approximately linear change in the coacervate phase polymer-micelle complex concentration $\phi_{p m}$.

\section{Micelle Bridging}

Micelle bridging occurs when segments along a polyelectrolyte chain are bound to two distinct micelle assemblies, forming a larger complex. Self-assembly in surfactant and polyelectrolyte solutions have been reported in the literature to form networks with nodes composed of self-assembled surfactant structures, with polyelectrolytes spanning the surfactant structures. ${ }^{64-66}$ The probability that a polyelectrolyte chain will bridge two micelle structures can be evaluated by considering the constrained partition function for the polyelectrolyte chain configurations that span two distinct micelle surface sites, relative to that of the entire set of unspecified chain configurations (i.e. the single-chain partition function $Q$ ). Each bridging configuration will have two segments along the chain bound to micelle surface sites, along with some number of salt bound sites $s_{b}$ in between, giving a total bridging segment length of $s_{b}+2$. Thus the bridging probability can be described as a function of concentrations $\phi_{p s}$ and $\phi_{p m}$, as well as the bridging segment length $s_{b}$. We consider inter-micelle bridging where the surface sites correspond to beads on distinct hard sphere chains in the micelle MC simulations, as well as intra-micelle bridging where the surface sites correspond to nonconsecutive beads on the same hard sphere chain. The micelle bridging probability can be described as follows, 
$P\left(\phi_{p m}, \phi_{p s}, s_{b}\right)=\sum_{\mathbf{r}} \sum_{\mathbf{r}^{\prime}} \frac{\sum_{s_{0}}^{N_{s}-2-s_{b}}\left[q\left(\mathbf{r}, s_{0}-1, M\right) \tilde{\Phi}\left(\mathbf{r}, \mathbf{r}^{\prime}, s_{b}\right) q\left(\mathbf{r}^{\prime}, N-s_{0}-1-s_{b}, M\right)\right]}{\sum_{s_{0}}^{N_{s}-2-s_{b}} Q}$

Here, $s_{0}$ is the location along the chain where the bridging segment begins, $\mathbf{r}$ and $\mathbf{r}^{\prime}$ are collocation points on the micelle surfaces, and the quantity $\tilde{\Phi}\left(\mathbf{r}, \mathbf{r}^{\prime}, s_{b}\right)$ corresponds to the Boltzmann weight of bridging from $\mathbf{r}$ to $\mathbf{r}^{\prime}$ in $s_{b}$ steps:

$$
\tilde{\Phi}\left(\mathbf{r}, \mathbf{r}^{\prime}, s_{b}\right)=\sum_{\left\{\mathbf{r}_{i}\right\}} \Phi\left(\mathbf{r}, M \mid \mathbf{r}_{0}, S\right)\left[\prod_{i}^{s_{b}} \Phi\left(\mathbf{r}_{i-1}, S \mid \mathbf{r}_{i}, S\right)\right] \Phi\left(\mathbf{r}_{s_{b}}, S \mid \mathbf{r}, M\right)
$$

The summation here is over all $r_{i}=r_{0}, r_{1}, \cdots, r_{s_{B}}$ positions of the bridging beads, and the quantities $\Phi\left(\mathbf{r}_{i-1}, \Psi_{i-1} \mid \mathbf{r}_{i}, \Psi_{i}\right)$ are once more the transition probability densities described earlier in this paper.

The micelle bridging probability was evaluated for a range of polymer-micelle complex concentrations $\left(0.12 \leq \phi_{p m} \leq 0.6\right)$, a range of polymer-salt complex concentrations $\left(1.25 \times 10^{-3} \leq \phi_{p s} \leq 1.875 \times 10^{-2}\right)$, and bridging segment lengths $s_{b}=1$ to $s_{b}=4$. Figure 6 a shows the bridging probability with a bridging segment length of $s_{b}=1$ as a function of polymer-micelle complex concentration $\phi_{p m}$, for varying values for $\phi_{p s}$. We initially focus on $s_{b}=1$ due to its predominance over $s_{b}>1$ (see later), and use it as an overall proxy for the frequency of bridging. The bridging probability for $\phi_{p s}=0.00125$ is significantly lower than in the higher $\phi_{p s}$ cases, which we attribute to the lack of available salt ions that are needed to bind the polymer to form the bridge. The bridging probabilities for the three higher $\phi_{p s}$ values are similar in magnitude to the low- $\phi_{p s}$ case in the low $\phi_{p m}$ limit, however exhibit a significant increase in bridging probability starting around $\phi_{p m}=0.35$ compared the low $\phi_{p s}$ case. This suggests that, away from the low- $\phi_{p s}$ limit, the ability for a micelle to bridge is limited by the availability and proximity of micelle surface sites to bridge rather than the availability of salt ions. We justify this by noting that as $\phi_{p m}$ is increased, the 

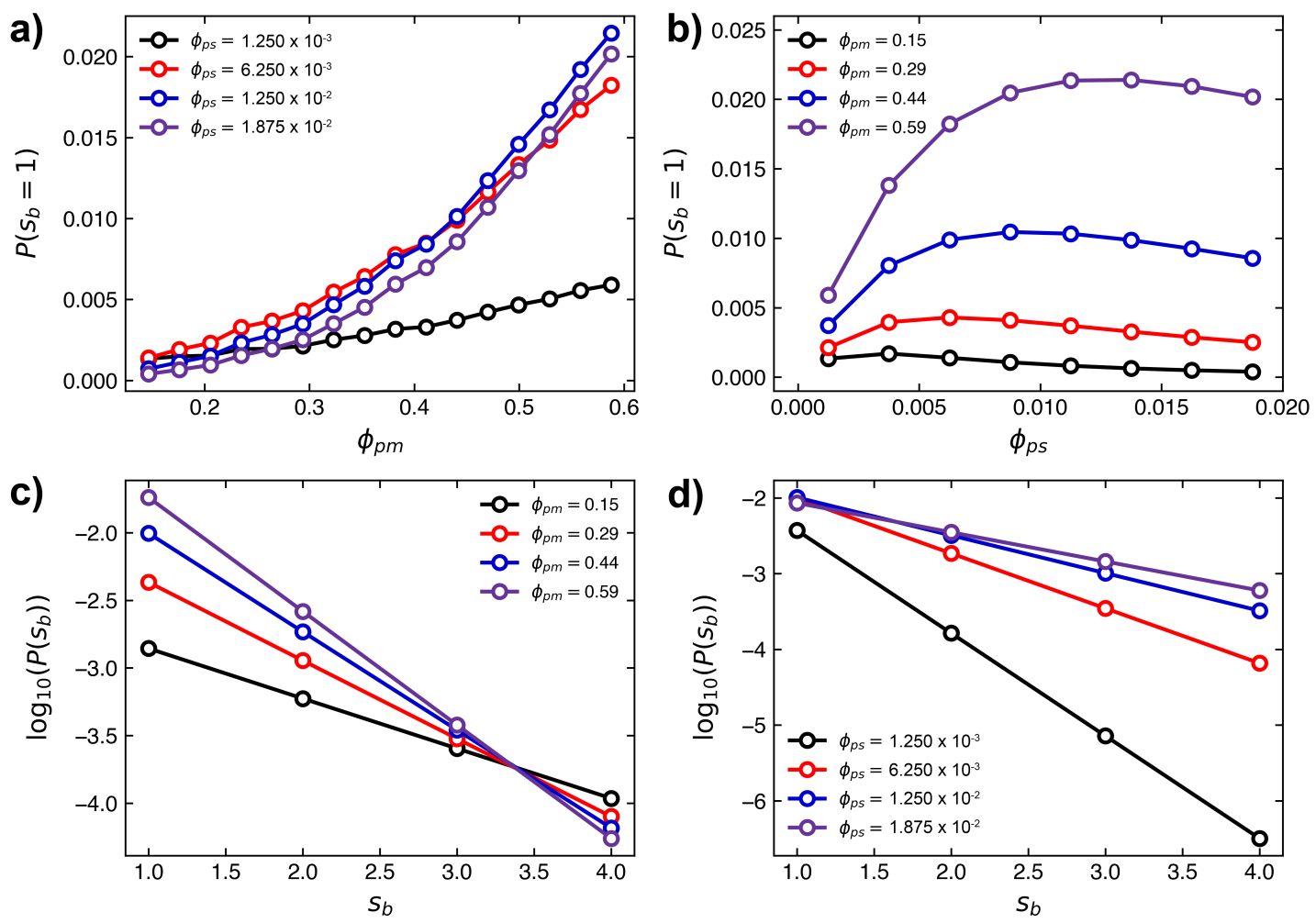

Figure 6: (a) Plot of the bridging probability $P$ for a segment with length $s_{b}=1$ vs. the polymer-micelle complex concentrations $\phi_{p m}$, while varying the polymer-salt complex concentration $\phi_{p s}$. (b) Plot of the bridging probability $P$ for a segment with length $s_{b}=1$ vs. the polymer-salt concentration, while varying the polymer-micelle complex concentration. (c) Plot of the logarithm of the bridging probability for a segment length $\log _{10}\left(P\left(s_{b}\right)\right)$, vs. the segment length $s_{b}$. The polymer-salt complex concentration is held constant at $\phi_{p s}=6.25 \times 10^{-3}$, and the polymer-micelle complex concentration is varied. (d) Plot of the logarithm of the bridging probability for a segment length $\log _{10}\left(P\left(s_{b}\right)\right)$, vs. the segment length $s_{b}$. The polymer-micelle complex concentration is held constant at $\phi_{p m}=6.25 \times 10^{-3}$, and the polymer-micelle complex concentration is varied. 
number of collocation points which correspond to a surface site of more than one micelle structure $\left(n_{s s}>1\right)$ is increased. These sites are expected to have the highest probability of bridging as the bridge origin and bridge terminus are located within the same collocation point.

Figure $6 \mathrm{~b}$ shows the bridging probability for a bridging segment length of $s_{b}=1$, as a function of polymer-salt complex concentration $\phi_{p s} . \phi_{p m}$ is varied corresponding to the different color plots. For all values of $\phi_{p m}$, the bridging probability increases as $\phi_{p s}$ is increased followed by a subtle decrease as $\phi_{p s}$ is further increased. The turnover point or the maxima of the plots are shifted towards higher $\phi_{p s}$ as $\phi_{p m}$ is increased. These results suggest that once there is sufficient available $\phi_{p s}$ to draw the polyelectrolyte off the micelle making it available to bridge, the limiting factor becomes the propensity for a bridging polyelectrolyte to terminate after a certain bridging length $s_{b}$.

Figure $6 \mathrm{c}$ and $6 \mathrm{~d}$ show the logarithm of the bridging probability as a function of bridging segment length $s_{b}$, with varying polymer-micelle complex concentrations (c) and polymersalt complex concentrations (d). In all cases, the logarithm of the bridging probability is linear with respect to $s_{b}$, indicating that the vast majority of bridges are $s_{b}=1$ justifying our previous use of this value as a proxy for bridging frequency. We expect the quantitative aspects of this trend to be sensitive to physical parameters, such as polymer length and stiffness, but note that the exponential decay of $P\left(s_{b}\right)$ is characteristic of a constant permonomer probability of bridging versus not bridging. This is observed over a wide range of values of $\phi_{p m}$ and $\phi_{p s}$. Interestingly, in Figure 6c, it is apparent that the relationship between bridging probability and $\phi_{p m}$ is reversed as $s_{b}$ is increased from $s_{b}=1$ to $s_{b}=4$. This implies that higher values of $\phi_{p m}$ lose more propensity to bridge as $s_{b}$ is increased than lower values of $\phi_{p m}$. This may be due to the fact that, while there are more surface-site collocation points at higher $\phi_{p m}$ that can form more bridges, there are also more available sites near the bridge origin increasing the probability of terminating the bridge at lower $s_{b}$.

Figure $6 \mathrm{~d}$ shows that for $s_{b}>1$, the bridging probability is monotonically increasing 
with increasing $\phi_{p s}$. In the low $\phi_{p s}$ limit, there is a steep drop in bridging probability as $s_{b}$ is increased, and as $\phi_{p s}$ is increased, this drop in bridging probability with increasing $s_{b}$ becomes less severe. This suggests that in the low $\phi_{p s}$ limit, the bridging is limited by the ability for the polyelectrolyte to come off the micelle to form a bridge origin due to a lack of available salt ions. In the high $\phi_{p s}$ region, the bridging probability for at a specific $s_{b}$ is limited by the propensity for the bridge to terminate at that value of $s_{b}$, and higher values $\phi_{p s}$ makes longer bridges more likely to form.
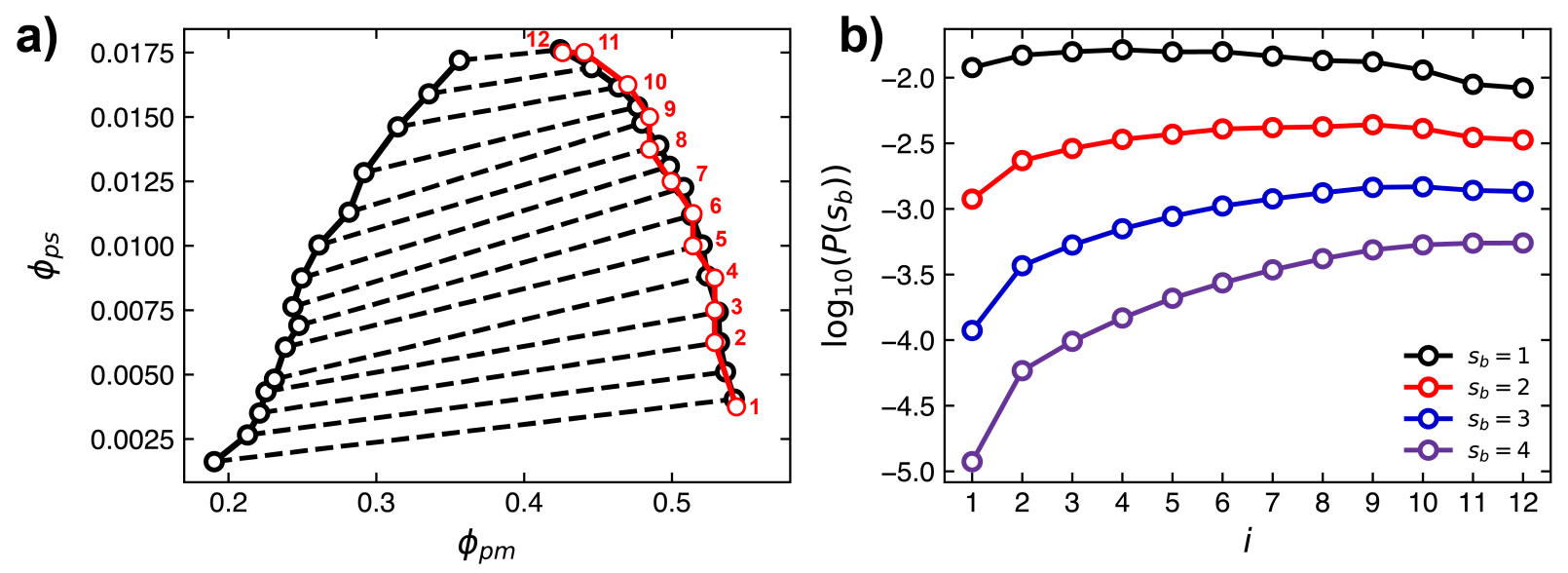

Figure 7: Plots showing a $\phi_{p s}$ versus $\phi_{p m}$ phase diagram (a) indicating a set of sampled coacervate-phase compositions (red) $i=1-12$. Phase diagram corresponds to a solution with $N_{p}=50, \tilde{\varepsilon}_{m}=-3.5$, and $\phi_{s s}=0.03$. (b) The logarithm of the bridging probability for a given bridge segment length $\log _{10}\left(P\left(s_{b}\right)\right)$ at the twelve sampled points $i$ in (a) for a number of different bridge segment lengths $s_{b}$.

The connection between micelle bridging and coacervation in polyelectrolyte and surfactant solutions is not well understood, but our model can provide some insights into the behavior of micelle bridging polyelectrolytes in the coacervate phase. In Figure 7a, the phase diagram for a system with parameters $N_{p}=50, \tilde{\varepsilon}_{m}=-3.5$, and $\phi_{s s}=0.03$; is shown in black. The MC simulation configurations corresponding to the complex concentrations closest to the values of the coacervate binodal are shown in red. The logarithm of the bridging probabilities for these points are shown in Figure $7 \mathrm{~b}$, where the $\mathrm{x}$-axis corresponds to the points $i$ along the coacervate binodal in 7 a. The points along the binodal are arranged in order of 
increasing $\phi_{p s}$ and are numbered 1 through 12. Figure 7b shows that the bridging probability for $s_{b}=1$ is relatively consistent through out the points along the binodal, though there is a slight decrease at large $i$ that we attribute to the decrease in micelle density $\phi_{p m}$. The points with lower value of $i$ and therefore lower amounts of $\phi_{p s}$ have lower bridging probabilities for $s_{b}>1$ when compared to higher values of $i$. This trend persists to higher $\phi_{p s}$ as $s_{b}$ is increased. These results indicate that, along the binodal in the direction of increasing $\phi_{p s}$, the number of short bridges remains relatively constant, however the propensity for longer bridges to form increases sharply.

\section{Conclusion}

We have developed a field theoretic description for solutions of polyelectrolytes with oppositelycharged surfactant micelles. In this model, self-consistent field theory is applied to consider polyelectrolytes in solution with worm-like surfactant micelles, whose structure is determined using Monte Carlo simulations. The compositional space is reduced by considering the electroneutral complexes formed between the polymer and surfactant-micelles, as well as the polymer and salt-ions. External applied potentials are informed by Monte Carlo simulations such that they correspond with the surfactant micelle structure, including the polymerbinding surface sites, and the polymer-excluding micelle interiors. The polyelectrolytes are treated as Gaussian chains, under the influence of these external potentials, where each segment is considered to be in one of two bound states: micelle-bound or salt ion-bound. The partition function for the polyelectrolytes in solution is evaluated numerically, from which free energy landscapes were developed in terms of the complex concentrations.

The free energy landscapes allow us to determine the binodals for the two-phase solutions, and phase diagrams were developed for the solutions with varying molecular parameters. These parameters included the degree of polymerization of the polyelectrolyte, the micelle surface-site concentration, and the micelle surface-site binding energy. We showed that 
correlation-driven, electrostatic attraction between the polyelectrolyte and surfactant-micelle heavily influence the coacervation behavior of the system. We observed that the extent of coacervation becomes saturated starting around $N_{p}=20$, and an increase in the coacervate phase macroion density was concomitant with an increase in the surface site volume fraction. Similarly, an increase in the magnitude of the polymer-micelle binding energy leads to a significant increase in the two-phase coexistence region.

This model allows us to consider the probability for having certain polyelectrolyte configurations; of particular interest were configurations that bridged micelle surface-sites that are distinct from one another. We determined the probabilities for polyelectrolyte chains to bridge two sites as a function of the bridge length and complex concentrations. The relationship between bridging and the concentrations of the complexes as well as the bridging length is not straightforward, though we found that at the low polymer-salt limit bridging is limited by the ability of the polymer to come off the micelle to start a bridge. We also found that with sufficient polymer-micelle concentration, and higher polymer-salt concentration, longer bridges become more probable. Bridging statistics at the compositions along the coacervate binodals are roughly constant as the compositions are varied, though in the low-polymer-salt limit bridging is suppressed, especially for the longer bridge lengths. While further investigation will be required to elucidate the connections between micelle bridging and coacervation in these systems, our results suggest micelle-bridging with short bridges (i.e. small $s_{b}$ ) is a feature of coacervation, and as the critical polymer-salt concentration is approached the formation of longer bridges may contribute to the transition into a single phase system.

In the development of this model, a few key assumptions were made in the interest of simplifying the model, though in principle many of these assumptions can be relaxed. One primary assumption is that the charges in the system are all paired with the opposite charge, a strong-binding limit approximation that is a reasonable starting point for high linearcharge density polyelectrolytes and high surface-charge density micelles. This simplifies 
our SCFT model to consider only two states $\Psi=S, M$, though generalization to more states is possible. ${ }^{99}$ We also limited our model to include only polymer-salt complexes and polymer-micelle complexes, inspired by the 'effective' ternary phase diagram schemes used by Svensson, et al. ${ }^{31,91}$ One direction for future work would be to extend beyond this limited parameter space, to provide predictions for a full five-component system of polycations, polyanions, cations, anions, and solvent. The other key assumption of our model is that the electrostatic binding energy felt by a polymer segment is doubled when it is adjacent to more than one micelle assembly. This is an ad hoc representation of more complicated charge correlation effects that are known to arise in nearby surfaces, ${ }^{100,101}$ and molecular predictions for the strength of this effect would be needed to parameterize this model for specific surfactant systems.

A key aspect of our model is the fact that the model incorporates the surfactant-micelle structure through external potential fields informed by Monte Carlo simulations. This means that any arbitrary structure can studied; while in this case we considered worm-like micelles, any structure can in principle be realized. This includes self-assembling micelle structures, and heterogeneously surface-charged structures such as patchy colloids or charged proteins. The relatively low computational costs of the model and its inherent versatility will allow us to continue to study phase behavior in mixed macroion systems, where the long-ranged electrostatics and the size disparities between the macroions can make traditional methods computationally expensive.

\section{Acknowledgement}

This material is based upon work supported by the National Science Foundation Graduate Research Fellowship Program under Grant No. DGE-1746047. The authors also acknowledge support by the National Science Foundation under Grant No. DMR-1654158 and the Sloan Minority PhD Program. We thank Dr. Tyler Lytle for providing valuable feedback and 
insight to this project.

\section{References}

(1) Mayya, K. S.; Bhattacharyya, A.; Argillier, J.-F. Micro-encapsulation by complex coacervation: Influence of surfactant. Polymer International 2003, 52, 644-647, DOI: 10.1002/pi.1125.

(2) Shulevich, Y. V.; Nguyen, T. H.; Tutaev, D. S.; Navrotskii, A. V.; Novakov, I. A. Purification of fat-containing wastewater using polyelectrolyte-surfactant complexes. Separation and Purification Technology 2013, 113, 18-23, DOI: $10.1016 / j$.seppur.2013.04.005.

(3) Singh, M.; Briones, M.; Ott, G.; O’Hagan, D. Cationic microparticles: a potent delivery system for DNA vaccines. Proceedings of the National Academy of Sciences 2000, 97, 811-816, DOI: 10.1073/pnas.97.2.811.

(4) Llamas, S.; Guzman, E.; Ortega, F.; Baghdadli, N.; Cazeneuve, C.; Rubio, R. G.; Luengo, G. S. Adsorption of polyelectrolytes and polyelectrolytes-surfactant mixtures at surfaces: a physico-chemical approach to a cosmetic challenge. Advances in colloid and interface science 2015, 222, 461-487, DOI: 10.1016/j.cis.2014.05.007.

(5) Bradbury, R.; Penfold, J.; Thomas, R. K.; Tucker, I. M.; Petkov, J. T.; Jones, C. Manipulating perfume delivery to the interface using polymer-surfactant interactions. Journal of colloid and interface science 2016, 466, 220-226, DOI: $10.1016 / j \cdot j$ cis. 2015.12 .041$.

(6) Bali, K.; Varga, Z.; Kardos, A.; Varga, I.; Gilányi, T.; Domján, A.; Wacha, A.; Bóta, A.; Mihály, J.; Mészáros, R. Effect of dilution on the nonequilibrium polyelectrolyte/surfactant association. Langmuir 2018, 34, 14652-14660, DOI: 10.1021/acs. langmuir.8b03255. 
(7) Kristen, N.; von Klitzing, R. Effect of polyelectrolyte/surfactant combinations on the stability of foam films. Soft Matter 2010, 6, 849-861, DOI: 10.1039/b917297a.

(8) Benhur, A. M.; Diaz, J.; Amin, S. Impact of polyelectrolyte-surfactant interactions on the rheology and wet lubrication performance of conditioning shampoo. International Journal of Cosmetic Science 2021, 43, 246-253, DOI: 10.1111/ics.12689.

(9) Bonnaud, M.; Weiss, J.; McClements, D. J. Interaction of a food-grade cationic surfactant (lauric arginate) with food-grade biopolymers (pectin, carrageenan, xanthan, alginate, dextran, and chitosan). Journal of agricultural and food chemistry 2010, 58, 9770-9777, DOI: 10.1021/jf101309h.

(10) Sharipova, A.; Aidarova, S.; Grigoriev, D.; Mutalieva, B.; Madibekova, G.; Tleuova, A.; Miller, R. Polymer-surfactant complexes for microencapsulation of vitamin E and its release. Colloids and Surfaces B: Biointerfaces 2016, 137, 152-157, DOI: $10.1016 / j . c o l s u r f b .2015 .03 .063$.

(11) Laquerbe, S.; Carvalho, A.; Schmutz, M.; Poirier, A.; Baccile, N.; Messaoud, G. B. pH-switchable pickering emulsions stabilized by polyelectrolyte-biosurfactant complex coacervate colloids. Journal of Colloid and Interface Science 2021, 600, 23-36, DOI: $10.1016 / j \cdot j$ jis. 2021.04 .135$.

(12) Thalberg, K.; Lindman, B.; Bergfeldt, K. Phase behavior of systems of polyacrylate and cationic surfactants. Langmuir 1991, 7, 2893-2898, DOI: 10.1021/1a00060a005.

(13) Khan, N.; Brettmann, B. Intermolecular interactions in polyelectrolyte and surfactant complexes in solution. Polymers 2019, 11, 51, DOI: 10.3390/polym11010051.

(14) Wang, Y.; Kimura, K.; Huang, Q.; Dubin, P. L.; Jaeger, W. Effects of salt on polyelectrolyte- micelle coacervation. Macromolecules 1999, 32, 7128-7134, DOI: 10.1021/ma990972v. 
(15) Hayakawa, K.; Kwak, J. C. Surfactant-polyelectrolyte interactions. 1. Binding of dodecyltrimethylammonium ions by sodium dextransulfate and sodium poly (styrenesulfonate) in aqueous solution in the presence of sodium chloride. The Journal of Physical Chemistry 1982, 86, 3866-3870, DOI: 10.1021/j100216a032.

(16) Hayakawa, K.; Kwak, J. C. Study of surfactant-polyelectrolyte interactions. 2. Effect of multivalent counterions on the binding of dodecyltrimethylammonium ions by sodium dextran sulfate and sodium poly (styrene sulfonate) in aqueous solution. The Journal of Physical Chemistry 1983, 87, 506-509, DOI: 10.1021/j100226a026.

(17) Malovikova, A.; Hayakawa, K.; Kwak, J. C. Surfactant-polyelectrolyte interactions. 4. Surfactant chain length dependence of the binding of alkylpyridinium cations to dextran sulfate. The Journal of Physical Chemistry 1984, 88, 1930-1933, DOI: $10.1021 / \mathrm{j} 150654 \mathrm{a} 002$.

(18) Maibaum, L.; Dinner, A. R.; Chandler, D. Micelle formation and the hydrophobic effect. The Journal of Physical Chemistry B 2004, 108, 6778-6781, DOI: 10.1021/jp037487t.

(19) López-López, M.; López-Cornejo, P.; Martín, V. I.; Ostos, F. J.; Checa-Rodríguez, C.; Prados-Carvajal, R.; Lebrón, J. A.; Huertas, P.; Moyá, M. L. Importance of hydrophobic interactions in the single-chained cationic surfactant-DNA complexation. Journal of colloid and interface science 2018, 521, 197-205, DOI: $10.1016 / j \cdot j$ jis. 2018.03 .048 .

(20) Chronakis, I. S.; Alexandridis, P. Rheological properties of oppositely charged polyelectrolyte- surfactant mixtures: effect of polymer molecular weight and surfactant architecture. Macromolecules 2001, 34, 5005-5018, DOI: 10.1021/ma000609k.

(21) Pagac, E. S.; Prieve, D. C.; Tilton, R. D. Kinetics and mechanism of cationic sur- 
factant adsorption and coadsorption with cationic polyelectrolytes at the silica- water interface. Langmuir 1998, 14, 2333-2342, DOI: 10.1021/la971308f.

(22) Chiappisi, L.; Hoffmann, I.; Gradzielski, M. Complexes of oppositely charged polyelectrolytes and surfactants-recent developments in the field of biologically derived polyelectrolytes. Soft Matter 2013, 9, 3896-3909, DOI: 10.1039/c3sm27698h.

(23) Jain, N.; Trabelsi, S.; Guillot, S.; McLoughlin, D.; Langevin, D.; Letellier, P.; Turmine, M. Critical aggregation concentration in mixed solutions of anionic polyelectrolytes and cationic surfactants. Langmuir 2004, 20, 8496-8503, DOI: 10.1021/1a0489918.

(24) Fuguet, E.; Ràfols, C.; Rosés, M.; Bosch, E. Critical micelle concentration of surfactants in aqueous buffered and unbuffered systems. Analytica Chimica Acta 2005, 548, 95-100, DOI: 10.1016/j.aca.2005.05.069.

(25) Kogej, K.; Škerjanc, J. Fluorescence and conductivity studies of polyelectrolyteinduced aggregation of alkyltrimethylammonium bromides. Langmuir 1999, 15, 42514258, DOI: $10.1021 /$ la9811517.

(26) Guillot, S.; McLoughlin, D.; Jain, N.; Delsanti, M.; Langevin, D. Polyelectrolytesurfactant complexes at interfaces and in bulk. Journal of Physics: Condensed Matter 2002, 15, S219, DOI: 10.1088/0953-8984/15/1/328.

(27) Wallin, T.; Linse, P. Polyelectrolyte-induced micellization of charged surfactants. Calculations based on a self-consistent field lattice model. Langmuir 1998, 14, 2940-2949, DOI: $10.1021 /$ la9712911.

(28) Li, D.; Kelkar, M. S.; Wagner, N. J. Phase behavior and molecular thermodynamics of coacervation in oppositely charged polyelectrolyte/surfactant systems: A cationic polymer JR 400 and anionic surfactant SDS mixture. Langmuir 2012, 28, 1034810362, DOI: $10.1021 / 1 a 301475$ s. 
(29) Messaoud, G. B.; Promeneur, L.; Brennich, M.; Roelants, S. L.; Le Griel, P.; Baccile, N. Complex coacervation of natural sophorolipid bolaamphiphile micelles with cationic polyelectrolytes. Green Chemistry 2018, 20, 3371-3385, DOI: 10.1039/c8gc01531g.

(30) Guillemet, F.; Piculell, L. Interactions in aqueous mixtures of hydrophobically modified polyelectrolyte and oppositely charged surfactant. Mixed micelle formation and associative phase separation. The Journal of Physical Chemistry 1995, 99, 9201-9209, DOI: $10.1021 / j 100022 \mathrm{a} 038$.

(31) Svensson, A.; Piculell, L.; Cabane, B.; Ilekti, P. A new approach to the phase behavior of oppositely charged polymers and surfactants. The Journal of Physical Chemistry B 2002, 106, 1013-1018, DOI: 10.1021/jp0120458.

(32) Van der Gucht, J.; Spruijt, E.; Lemmers, M.; Stuart, M. A. C. Polyelectrolyte complexes: Bulk phases and colloidal systems. Journal of colloid and interface science 2011, 361, 407-422, DOI: 10.1016/j.jcis.2011.05.080.

(33) Perry, S. L.; Li, Y.; Priftis, D.; Leon, L.; Tirrell, M. The effect of salt on the complex coacervation of vinyl polyelectrolytes. Polymers 2014, 6, 1756-1772, DOI: $10.3390 /$ polym6061756.

(34) Priftis, D.; Xia, X.; Margossian, K. O.; Perry, S. L.; Leon, L.; Qin, J.; de Pablo, J. J.; Tirrell, M. Ternary, tunable polyelectrolyte complex fluids driven by complex coacervation. Macromolecules 2014, 47, 3076-3085, DOI: 10.1021/ma500245j.

(35) Lee, J.; Popov, Y. O.; Fredrickson, G. H. Complex coacervation: A field theoretic simulation study of polyelectrolyte complexation. The Journal of chemical physics 2008, 128, 224908, DOI: 10.1063/1.2936834.

(36) Sing, C. E. Development of the modern theory of polymeric complex coac- 
ervation. Advances in colloid and interface science 2017, 239, 2-16, DOI: $10.1016 / j . \operatorname{cis} .2016 .04 .004$.

(37) Lytle, T. K.; Sing, C. E. Transfer matrix theory of polymer complex coacervation. Soft Matter 2017, 13, 7001-7012, DOI: 10.1039/c7sm01080j.

(38) Perry, S. L.; Sing, C. E. Prism-based theory of complex coacervation: Excluded volume versus chain correlation. Macromolecules 2015, 48, 5040-5053, DOI: 10.1021/acs.macromol.5b01027.

(39) Adhikari, S.; Leaf, M. A.; Muthukumar, M. Polyelectrolyte complex coacervation by electrostatic dipolar interactions. The Journal of chemical physics 2018, 149, 163308, DOI: $10.1063 / 1.5029268$.

(40) Zhang, P.; Shen, K.; Alsaifi, N. M.; Wang, Z.-G. Salt partitioning in complex coacervation of symmetric polyelectrolytes. Macromolecules 2018, 51, 5586-5593, DOI: 10.1021/acs.macromol. 8 b00726.

(41) Borue, V. Y.; Erukhimovich, I. Y. A statistical theory of globular polyelectrolyte complexes. Macromolecules 1990, 23, 3625-3632, DOI: 10.1021/ma00217a015.

(42) Li, L.; Srivastava, S.; Andreev, M.; Marciel, A. B.; de Pablo, J. J.; Tirrell, M. V. Phase behavior and salt partitioning in polyelectrolyte complex coacervates. Macromolecules 2018, 51, 2988-2995, DOI: 10.1021/acs.macromol.8b00238.

(43) Sing, C.; Perry, S. Recent progress in the science of complex coacervation. Soft Matter 2020, 16, DOI: 10.1039/D0SM00001A, DOI: 10.1039/d0sm00001a.

(44) Salehi, A.; Larson, R. G. A Molecular Thermodynamic Model of Complexation in Mixtures of Oppositely Charged Polyelectrolytes with Explicit Account of Charge Association/Dissociation. Macromolecules 2016, 49, 9706-9719, DOI: 10.1021/acs.macromol. 6b01464. 
(45) Friedowitz, S.; Salehi, A.; Larson, R. G.; Qin, J. Role of electrostatic correlations in polyelectrolyte charge association. J. Chem. Phys. 2018, 149, 163335, DOI: $10.1063 / 1.5034454$.

(46) Lou, J.; Friedowitz, S.; Qin, J.; Xia, Y. Tunable coacervation of well-defined homologous polyanions and polycations by local polarity. ACS Cent. Sci. 2019, 5, 549-557, DOI: $10.1021 /$ acscentsci.8b00964.

(47) Qin, J.; de Pablo, J. J. Criticality and connectivity in macromolecular charge complexation. Macromolecules 2016, 49, 8789-8800, DOI: 10.1021/acs.macromol.6b02113.

(48) Priftis, D.; Tirrell, M. Phase behaviour and complex coacervation of aqueous polypeptide solutions. Soft Matter 2012, 8, 9396-9405, DOI: 10.1039/c2sm25604e.

(49) Danielsen, S. P. O.; McCarty, J.; Shea, J.-E.; Delaney, K. T.; Fredrickson, G. H. Molecular design of self-coacervation phenomena in block polyampholytes. Proc. Natl. Acad. Sci. U. S. A. 2019, 8224-8232, DOI: 10.1073/pnas.1900435116.

(50) Delaney, K. T.; Fredrickson, G. H. Theory of polyelectrolyte complexation - Complex coacervates are self-coacervates. J. Chem. Phys. 2017, 146, 224902, DOI: $10.1063 / 1.4985568$.

(51) Marciel, A. B.; Srivastava, S.; Tirrell, M. V. Structure and rheology of polyelectrolyte complex coacervates. Soft Matter 2018, DOI: 10.1039/C7SM02041D, DOI: 10.1039/c7sm02041d.

(52) Srivastava, S.; Andreev, M.; Levi, A. E.; Goldfeld, D. J.; Mao, J.; Heller, W. T.; de Pablo, J. J.; Tirrell, M. V. Gel phase formation in dilute triblock copolyelectrolyte complexes. Nat. Commun. 2017, 8, 14131, DOI: 10.1038/ncomms14131.

(53) Huang, J.; Morin, F.; Laaser, J. E. Charge-Density-Dominated Phase Behavior and 
Viscoelasticity of Polyelectrolyte Complex Coacervates. Macromolecules 2019, 52, 4957-4967, DOI: 10.1021/acs.macromol.9b00036.

(54) Liu, Y.; Santa Chalarca, C.; Carmean, R.; Olson, R.; Madinya, J.; Sumerlin, B.; Sing, C.; Emrick, T.; Perry, S. Effect of polymer chemistry on the linear viscoelasticity of complex coacervates. Macromolecules 2020, 53, 7851-7864, DOI: 10.1021/acs.macromol.0c00758.

(55) Li, Y.; Dubin, P. L.; Havel, H. A.; Edwards, S. L.; Dautzenberg, H. Complex formation between polyelectrolyte and oppositely charged mixed micelles: soluble complexes vs coacervation. Langmuir 1995, 11, 2486-2492, DOI: 10.1021/1a00007a029.

(56) De Kruif, C. G.; Weinbreck, F.; de Vries, R. Complex coacervation of proteins and anionic polysaccharides. Current opinion in colloid \& interface science 2004, 9, 340349, DOI: $10.1016 /$ j.cocis. 2004.09.006.

(57) Obermeyer, A. C.; Mills, C. E.; Dong, X.-H.; Flores, R. J.; Olsen, B. D. Complex coacervation of supercharged proteins with polyelectrolytes. Soft Matter 2016, 12, 3570-3581, DOI: 10.1039/c6sm00002a.

(58) Ghorbani Gorji, E.; Waheed, A.; Ludwig, R.; Toca-Herrera, J. L.; Schleining, G.; Ghorbani Gorji, S. Complex coacervation of milk proteins with sodium alginate. Journal of agricultural and food chemistry 2018, 66, 3210-3220, DOI: 10.1021/acs. jafc.7b03915.

(59) Antonietti, M.; Conrad, J.; Thuenemann, A. Polyelectrolyte-surfactant complexes: a new type of solid, mesomorphous material. Macromolecules 1994, 27, 6007-6011, DOI: $10.1021 / \mathrm{ma} 00099 \mathrm{a} 011$.

(60) Ober, C. K.; Wegner, G. Polyelectrolyte-Surfactant Complexes in the Solid State: Facile building blocks for self-organizing materials. Advanced Materials 1997, 9, 1731, DOI: $10.1002 /$ adma. 19970090104. 
(61) Lapitsky, Y.; Kaler, E. W. Formation of surfactant and polyelectrolyte gel particles in aqueous solutions. Colloids and Surfaces A: Physicochemical and Engineering Aspects 2004, 250, 179-187, DOI: 10.1016/j.colsurfa.2004.05.028.

(62) Lapitsky, Y.; Kaler, E. W. Surfactant and polyelectrolyte gel particles for encapsulation and release of aromatic oils. Soft Matter 2006, 2, 779-784, DOI: 10.1039/b607910e.

(63) Bergström, L. M.; Kjellin, U. M.; Claesson, P. M.; Grillo, I. Small-angle neutron scattering study of mixtures of cationic polyelectrolyte and anionic surfactant: Effect of polyelectrolyte charge density. The Journal of Physical Chemistry B 2004, 108, 1874-1881, DOI: 10.1021/jp036562x.

(64) Gradzielski, M.; Hoffmann, I. Polyelectrolyte-surfactant complexes (PESCs) composed of oppositely charged components. Current Opinion in Colloid $\mathscr{E}$ Interface Science 2018, 35, 124-141, DOI: 10.1016/j.cocis.2018.01.017.

(65) Lam, V. D.; Walker, L. M. A pH-induced transition of Surfactant- polyelectrolyte aggregates from cylindrical to string-of-pearls structure. Langmuir 2010, 26, 10489 10496, DOI: $10.1021 /$ la100130v.

(66) Goswami, M.; Borreguero, J. M.; Pincus, P. A.; Sumpter, B. G. Surfactant-mediated polyelectrolyte self-assembly in a polyelectrolyte-surfactant complex. Macromolecules 2015, 48, 9050-9059, DOI: 10.1021/acs.macromol.5b02145.

(67) Hervé, P.; Destarac, M.; Berret, J.-F.; Lal, J.; Oberdisse, J.; Grillo, I. Novel coreshell structure for colloids made of neutral/polyelectrolyte diblock copolymers and oppositely charged surfactants. EPL (Europhysics Letters) 2002, 58, 912, DOI: 10.1209/epl/i2002-00104-y.

(68) Dubin, P. L.; Curran, M. E.; Hua, J. Critical linear charge density for binding of a 
weak polycation to an anionic/nonionic mixed micelle. Langmuir 1990, 6, 707-709, DOI: $10.1021 / 1$ a00093a030.

(69) Kizilay, E.; Kayitmazer, A. B.; Dubin, P. L. Complexation and coacervation of polyelectrolytes with oppositely charged colloids. Advances in colloid and interface science 2011, 167, 24-37, DOI: $10.1016 /$ j.cis.2011.06.006.

(70) Manning, G. S. Limiting laws and counterion condensation in polyelectrolyte solutions I. Colligative properties. The journal of chemical Physics 1969, 51, 924-933, DOI: 10.1063/1.1672157.

(71) Ou, Z.; Muthukumar, M. Entropy and enthalpy of polyelectrolyte complexation: Langevin dynamics simulations. The Journal of chemical physics 2006, 124, 154902, DOI: $10.1063 / 1.2178803$.

(72) Chang, L.-W.; Lytle, T. K.; Radhakrishna, M.; Madinya, J. J.; Vélez, J.; Sing, C. E.; Perry, S. L. Sequence and entropy-based control of complex coacervates. Nature communications 2017, 8, 1-8, DOI: 10.1038/s41467-017-01249-1.

(73) Madinya, J. J.; Chang, L.-W.; Perry, S. L.; Sing, C. E. Sequence-dependent selfcoacervation in high charge-density polyampholytes. Molecular Systems Design 83 Engineering 2020, 5, 632-644, DOI: 10.1039/c9me00074g.

(74) Kudlay, A.; Ermoshkin, A. V.; Olvera de La Cruz, M. Complexation of oppositely charged polyelectrolytes: effect of ion pair formation. Macromolecules 2004, 37, 92319241, DOI: 10.1021/ma048519t.

(75) Kudlay, A.; Olvera de la Cruz, M. Precipitation of oppositely charged polyelectrolytes in salt solutions. J. Chem. Phys. 2004, 120, 404-412, DOI: 10.1063/1.1629271.

(76) Castelnovo, M.; Joanny, J.-F. Complexation between oppositely charged polyelec- 
trolytes: Beyond the Random Phase Approximation. The European Physical Journal E 2001, 6, 377-386, DOI: 10.1007/s10189-001-8051-7.

(77) Kayitmazer, A. B. Thermodynamics of complex coacervation. Advances in colloid and interface science 2017, 239, 169-177, DOI: 10.1016/j.cis.2016.07.006.

(78) Kumar, A.; Dubin, P. L.; Hernon, M. J.; Li, Y.; Jaeger, W. Temperature-Dependent Phase Behavior of Polyelectrolyte- Mixed Micelle Systems. The Journal of Physical Chemistry B 2007, 111, 8468-8476, DOI: 10.1021/jp067919a.

(79) Wang, Y.; Kimura, K.; Dubin, P. L.; Jaeger, W. Polyelectrolyte- micelle coacervation: Effects of micelle surface charge density, polymer molecular weight, and polymer/surfactant ratio. Macromolecules 2000, 33, 3324-3331, DOI: 10.1021/ma991886y.

(80) Pandav, G.; Pryamitsyn, V.; Errington, J.; Ganesan, V. Multibody Interactions, Phase Behavior, and Clustering in Nanoparticle-Polyelectrolyte Mixtures. The Journal of Physical Chemistry B 2015, 119, 14536-14550, DOI: 10.1021/acs.jpcb.5b07905.

(81) Samanta, R.; Halabe, A.; Ganesan, V. Influence of charge regulation and charge heterogeneity on complexation between polyelectrolytes and proteins. The Journal of Physical Chemistry B 2020, 124, 4421-4435, DOI: 10.1021/acs.jpcb.0c02007.

(82) Samanta, R.; Ganesan, V. Direct Simulations of Phase Behavior of Mixtures of Oppositely Charged Proteins/Nanoparticles and Polyelectrolytes. The Journal of Physical Chemistry B 2020, 124, 10943-10951, DOI: 10.1021/acs.jpcb.0c08317.

(83) Samanta, R.; Ganesan, V. Influence of protein charge patches on the structure of protein-polyelectrolyte complexes. Soft Matter 2018, 14, 9475-9488, DOI: $10.1039 / \mathrm{c} 8 \mathrm{sm} 01535 \mathrm{j}$. 
(84) Pryamitsyn, V.; Ganesan, V. Interplay between depletion and electrostatic interactions in polyelectrolyte-nanoparticle systems. Macromolecules 2014, 47, 6095-6112, DOI: $10.1021 / \mathrm{ma} 501014 \mathrm{u}$.

(85) Rathee, V. S.; Sidky, H.; Sikora, B. J.; Whitmer, J. K. Role of associative charging in the entropy-energy balance of polyelectrolyte complexes. J. Am. Chem. Soc. 2018, 140, 15319-15328, DOI: 10.1021/jacs.8b08649.

(86) Neitzel, A. E.; Fang, Y. N.; B, Y.; M, R. A.; de Pablo, J.; Tirrell, M. V. Polyelectrolyte complex coacervation across a broad range of charge densities. Macromolecules $\mathbf{2 0 2 1}$, 54, 6878-6890, DOI: 10.1021/acs.macromol.1c00703.

(87) Lytle, T. K.; Sing, C. E. Tuning chain interaction entropy in complex coacervation using polymer stiffness, architecture, and salt valency. Molecular Systems Design $\&$ Engineering 2018, 3, 183-196, DOI: 10.1039/c7me00108h.

(88) Lytle, T. K.; Chang, L.-W.; Markiewicz, N.; Perry, S. L.; Sing, C. E. Designing electrostatic interactions via polyelectrolyte monomer sequence. ACS central science $\mathbf{2 0 1 9}$, 5, 709-718, DOI: 10.1021/acscentsci.9b00087.

(89) Lytle, T. K.; Salazar, A. J.; Sing, C. E. Interfacial properties of polymeric complex coacervates from simulation and theory. The Journal of chemical physics 2018, 149 , 163315, DOI: $10.1063 / 1.5029934$.

(90) Knoerdel, A. R.; Blocher McTigue, W. C.; Sing, C. E. Transfer matrix model of pH effects in polymeric complex coacervation. The Journal of Physical Chemistry B 2021, 125, 8965-8980, DOI: 10.1021/acs.jpcb.1c03065.

(91) Svensson, A.; Norrman, J.; Piculell, L. Phase behavior of polyion- surfactant ion complex salts: Effects of surfactant chain length and polyion length. The Journal of Physical Chemistry B 2006, 110, 10332-10340, DOI: 10.1021/jp057402j. 
(92) Thalberg, K.; Lindman, B.; Karlstroem, G. Phase behavior of systems of cationic surfactant and anionic polyelectrolyte: influence of surfactant chain length and polyelectrolyte molecular weight. The Journal of Physical Chemistry 1991, 95, 3370-3376, DOI: $10.1021 / \mathrm{j} 100161 \mathrm{a} 073$.

(93) Wallin, T.; Linse, P. Monte Carlo simulations of polyelectrolytes at charged micelles. 3. Effects of surfactant tail length. The Journal of Physical Chemistry B 1997, 101, 5506-5513, DOI: 10.1021/jp970224c.

(94) Liu, J.; Takisawa, N.; Shirahama, K.; Abe, H.; Sakamoto, K. Effect of Polymer Size on the Polyelectrolyte- Surfactant Interaction. The Journal of Physical Chemistry B 1997, 101, 7520-7523, DOI: 10.1021/jp9711981.

(95) Singh, S. K.; Nilsson, S. Thermodynamics of interaction between some cellulose ethers and SDS by titration microcalorimetry: II. Effect of polymer hydrophobicity. Journal of colloid and interface science 1999, 213, 152-159, DOI: https://doi.org/10.1006/jcis.1999.6121.

(96) Zhou, S.; Burger, C.; Yeh, F.; Chu, B. Charge density effect of polyelectrolyte chains on the nanostructures of polyelectrolyte- surfactant complexes. Macromolecules 1998, 31, 8157-8163, DOI: 10.1021/ma9810058.

(97) Wang, C.; Tam, K. Interaction between polyelectrolyte and oppositely charged surfactant: effect of charge density. The Journal of Physical Chemistry B 2004, 108, 8976-8982, DOI: 10.1021/jp049647m.

(98) Jonsson, M.; Linse, P. Polyelectrolyte-macroion complexation. I. Effect of linear charge density, chain length, and macroion charge. The Journal of Chemical Physics 2001, 115, 3406-3418, DOI: 10.1063/1.1385792.

(99) Sing, C. E. Micro- to macro-phase separation transition in sequence-defined coacervates. J. Chem. Phys. 2020, 152, DOI: 10.1063/1.5140756, DOI: 10.1063/1.5140756. 
(100) Moreira, A. G.; Netz, R. R. Binding of similarly charged plates with counterions only. Physical review letters 2001, 87, 078301, DOI: 10.1103/physrevlett.87.078301.

(101) Netz, R. R. Electrostatistics of counter-ions at and between planar charged walls: From Poisson-Boltzmann to the strong-coupling theory. The European Physical Journal E 2001, 5, 557-574, DOI: 10.1007/s101890170039.

(102) Lauw, Y.; Leermakers, F. A. M.; Cohen Stuart, M. Persistence Length of Wormlike Micelles Composed of Ionic Surfactants: Self-Consistent-Field Predictions. J. Phys. Chem. B 2007, 111, 8158-8168, DOI: 10.1021/jp071756o.

(103) Fredrickson, G., et al. The equilibrium theory of inhomogeneous polymers; Oxford University Press on Demand, 2006; Vol. 134.

(104) Doi, M.; Edwards, S. F. The theory of polymer dynamics; oxford university press, 1988; Vol. 73.

(105) Carnahan, N. F.; Starling, K. E. Equation of State for Nonattracting Rigid Spheres. The Journal of Chemical Physics 1969, 51, 635-636, DOI: 10.1063/1.1672048.

(106) Johnston, B. M.; Johnston, C. W.; Letteri, R. A.; Lytle, T. K.; Sing, C. E.; Emrick, T.; Perry, S. L. The effect of comb architecture on complex coacervation. Organic \& biomolecular chemistry 2017, 15, 7630-7642, DOI: 10.1039/c7ob01314k.

(107) Spruijt, E.; Westphal, A. H.; Borst, J. W.; Cohen Stuart, M. A.; van der Gucht, J. Binodal compositions of polyelectrolyte complexes. Macromolecules 2010, 43, 64766484, DOI: $10.1021 / \mathrm{ma101031t.}$

(108) Radhakrishna, M.; Basu, K.; Liu, Y.; Shamsi, R.; Perry, S. L.; Sing, C. E. Molecular connectivity and correlation effects on polymer coacervation. Macromolecules 2017, 50, 3030-3037, DOI: 10.1021/acs.macromol.6b02582. 


\section{TOC Graphic}

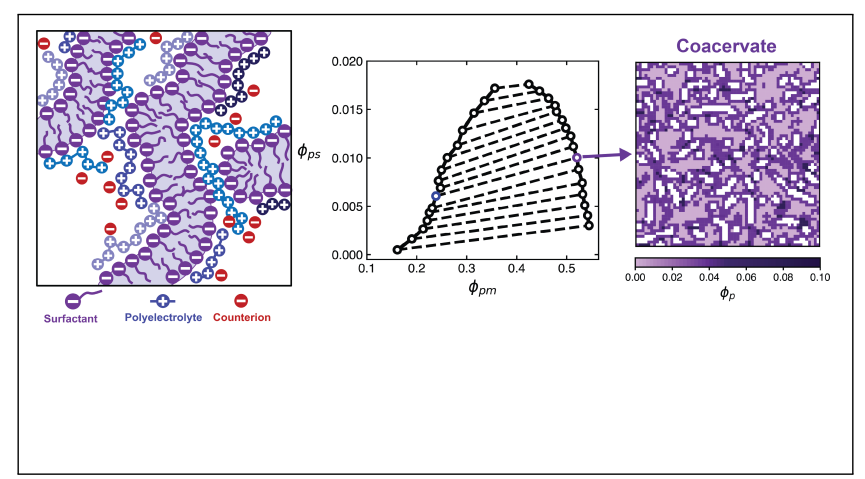

